\title{
Bacterial Curli Protein Promotes the Conversion of PAP(248-286) into the Amyloid SEVI: Cross-Seeding of Dissimilar Amyloid Sequences
}

Fragments of prostatic acid phosphatase ( $\left.\mathrm{PAP}_{248-286}\right)$ in human semen dramatically increase HIV infection efficiency by increasing virus adhesion to target cells. $\mathrm{PAP}_{248-286}$ only enhances HIV infection in the form of amyloid aggregates termed SEVI (Semen Enhancer of Viral Infection), however monomeric $\mathrm{PAP}_{248-286}$ aggregates very slowly in isolation. It has therefore been suggested that SEVI fiber formation in vivo may be promoted by exogenous factors. We show here that a bacterially-produced extracellular amyloid (curli or Csg) acts as a catalytic agent for SEVI formation from $\mathrm{PAP}_{248-286}$ at low concentrations in vitro, producing fibers that retain the ability to enhance HIV ( Human Immunodeficiency Virus) infection. Kinetic analysis of the cross-seeding effect shows an unusual pattern. Cross-seeding $\mathrm{PAP}_{248-286}$ with curli only moderately affects the nucleation rate while significantly enhancing the growth of fibers from existing nuclei. This pattern is in contrast to most previous observations of cross-seeding, which show cross-seeding partially bypasses the nucleation step but has little effect on fiber elongation. Seeding other amyloidogenic proteins (IAPP (islet amyloid polypeptide) and $A \beta_{1-40}$ ) with curli showed varied results. Curli cross-seeding decreased the lag-time of IAPP amyloid formation but strongly inhibited IAPP elongation. Curli cross-seeding exerted a complicated concentration dependent effect on $A \beta_{1-40}$ fibrillogenesis kinetics. Combined, these results suggest that the interaction of amyloidogenic proteins with preformed fibers of a different type can take a variety of forms and is not limited to epitaxial nucleation between proteins of similar sequence. The ability of curli fibers to interact with proteins of dissimilar sequences suggests cross-seeding may be a more general phenomenon than previously supposed. 


\section{Bacterial Curli Protein Promotes the Conversion of PAP(248-286) into the Amyloid SEVI: Cross-Seeding of Dissimilar Amyloid Sequences}

Kevin Hartman, ${ }^{\text {a,b }}$ Jeffrey R. Brender, ${ }^{\text {a,b }}$ Kazuaki Monde, ${ }^{c, 1}$ Akira Ono, ${ }^{c}$ Margery L. Evans, ${ }^{\text {d Nataliya }}$ Popovych, ${ }^{\mathrm{a}, \mathrm{b}, 2}$ Matthew R. Chapman, ${ }^{\mathrm{d}}$ Ayyalusamy Ramamoorthy ${ }^{\mathrm{a}, \mathrm{b}}$

${ }^{a}$ Departments of Chemistry, ${ }^{b}$ Biophysics, and ${ }^{d}$ Molecular, Cellular, and Developmental Biology, University of Michigan, Ann Arbor, MI 48109-1055, USA.

${ }^{\mathrm{c}}$ Department of Microbiology and Immunology, University of Michigan Medical School, MI 48109-1055, USA..

${ }^{1}$ Present Address: Department of Medical Virology, Faculty of Life Sciences, Kumamoto University, Kumamoto 860-0811 Japan.

${ }^{2}$ Present Address: Genentech, 1 DNA Way South San Francisco, CA 94080.

Corresponding Author: Ayyalusamy Ramamoorthy, ramamoor@umich.edu, 930 North University, Ann Arbor, MI 48109-1055, USA.

Keywords: functional amyloid, strain, HIV, kinetics, seeding 
ABSTRACT: Fragments of prostatic acid phosphatase $\left(\mathrm{PAP}_{248-286}\right)$ in human semen dramatically increase HIV infection efficiency by increasing virus adhesion to target cells. $\mathrm{PAP}_{248-286}$ only enhances HIV infection in the form of amyloid aggregates termed SEVI (Semen Enhancer of Viral Infection), however monomeric $\mathrm{PAP}_{248-286}$ aggregates very slowly in isolation. It has therefore been suggested that SEVI fiber formation in vivo may be promoted by exogenous factors. We show here that a bacteriallyproduced extracellular amyloid (curli or Csg) acts as a catalytic agent for SEVI formation from PAP 248 286 at low concentrations in vitro, producing fibers that retain the ability to enhance HIV (Human Immunodeficiency Virus) infection. Kinetic analysis of the cross-seeding effect shows an unusual pattern. Cross-seeding $\mathrm{PAP}_{248-286}$ with curli only moderately affects the nucleation rate while significantly enhancing the growth of fibers from existing nuclei. This pattern is in contrast to most previous observations of cross-seeding, which show cross-seeding partially bypasses the nucleation step but has little effect on fiber elongation. Seeding other amyloidogenic proteins (IAPP (islet amyloid polypeptide) and $A \beta_{1-40}$ ) with curli showed varied results. Curli cross-seeding decreased the lag-time of IAPP amyloid formation but strongly inhibited IAPP elongation. Curli cross-seeding exerted a complicated concentration dependent effect on $A \beta_{1-40}$ fibrillogenesis kinetics. Combined, these results suggest that the interaction of amyloidogenic proteins with preformed fibers of a different type can take a variety of forms and is not limited to epitaxial nucleation between proteins of similar sequence. The ability of curli fibers to interact with proteins of dissimilar sequences suggests cross-seeding may be a more general phenomenon than previously supposed.

\section{INTRODUCTION}

Over 33 million people worldwide are currently infected with the HIV virus. While HIV is readily transmitted in vivo, HIV is a surprisingly ineffective pathogen in vitro.(1) The key barrier to effective viral transmission is attachment to the target cell surface. The short-half life of almost all viruses in solution ( 6 hours for HIV) ensures that infection can only be achieved only if a significant number of viral particles adhere to the cell surface in a relatively narrow time window upon exposure.(2) A peptide fragment naturally occurring in human seminal fluid $\left(\mathrm{PAP}_{248-286}\right)$ facilitates this first step, and in doing so, greatly enhances the infectivity of HIV.(3) When fibrillized into amyloid fibers termed SEVI (Semen-derived Enhancer of Virus Infection),(3-5) PAP $248-286$ bridges the membranes of the HIV virion and target cells. $(3,6-9)$ The result is a dramatic increase in the infectivity of the HIV virus. While up to 100,000 HIV virions are required to establish infection in the absence of SEVI amyloid fibers, only 3 are needed in its presence.(3)

This enhancement is specifically linked to the SEVI amyloid conformation of $\mathrm{PAP}_{248-286}$ as monomers of $\mathrm{PAP}_{248-286}$ have little effect. $(3,7,10,11)$ Since $\mathrm{PAP}_{248-286}$ only enhances HIV infection when in the aggregated SEVI amyloid form, molecules that initiate fibrillization of $\mathrm{PAP}_{248-286}$ can indirectly increase HIV infectivity. While amyloid formation is typically energetically favorable, the actual rate of formation can be very slow. Specifically, amyloid formation is a nucleation-dependent process in which a slow rate-limiting nucleation step is followed by the faster process of extension of the fiber from the nuclei. $\mathrm{PAP}_{248-286}$ is subject to significant proteolytic degradation in seminal plasma unless it is in the SEVI amyloid form.(12) The total production of SEVI is therefore ultimately controlled by the rate of amyloidosis due to the proteolytic degradation of unfibrillized $\mathrm{PAP}_{248-286}$. $\mathrm{PAP}_{248-286}$ is very slow to fibrillize in the absence of shaking in vitro, $(13,14)$ which suggests SEVI production will be very limited in vivo. Nevertheless, the SEVI amyloid form is found in semen at concentrations of up to $35 \mu \mathrm{g} / \mathrm{mL}$. $(3,9)$ This finding suggests additional cofactors may be present that accelerate SEVI formation from $\mathrm{PAP}_{248-286}$ before the $\mathrm{PAP}_{248-286}$ monomer is degraded by the cell's proteolytic machinery.(14) 
One set of likely cofactors are other cellular proteins, since the factors that drive self-association of amyloidogenic proteins also tend to favor promiscuous binding to a variety of other proteins. $(15,16)$ In particular, amyloidogenic proteins frequently have the ability to cross-polymerize, that is amyloid fibers from one protein can catalyze the formation of amyloid fibers from another amyloidogenic protein. Cross-seeding amyloid fibrillogenesis in this manner can dramatically enhance the kinetics of amyloid formation by providing preformed nuclei for further aggregation. Furthermore, the final amyloid fiber can in some cases takes on some of the characteristics of fibers from the initial seeding amyloid protein.(17-19)

Amyloid nucleation is often considered a highly specific process dependent on a high degree of similarity in both peptide sequence and fiber morphology between the seed and the protein being nucleated. This conclusion has largely been motivated by research on prion amyloid fibers, where the inability of highly homologous prions to cross-seed amyloid formation presents an inter-species barrier to prion transmission. $(20,21)$ However, this view has recently been challenged by the observation of efficient cross-polymerization between several non-prion amyloid species. (22-27) Seeds formed from amyloid fibers of some non-homologous proteins can either reduce or eliminate the lag-time of amyloid formation of another protein, $(22-26,28)$ although the phenomenon is not universal and even single point mutations have been shown to disrupt cross-seeding in some cases.(26, 29-31) Cross-seeding may also be asymmetric, with one protein able to seed another but not be seeded by it. $(32,33)$

The amyloids produced by many bacteria and fungi are of particular interest in considering possible cross-seeding reactions with $\mathrm{PAP}_{248-286}$ because of the high incidence of microbial infection in the vagina and the frequent coexistence with bacterial and fungal infection with HIV infection.(34) A particularly well studied example is a highly amyloidogenic protein secreted by Escherichia coli (E. Coli) and related bacteria, CsgA, that polymerizes into curli fibers that are involved in cell-to-cell and cell-to-surface interactions.(35, 36) Although curli fibers have not to our knowledge been directly detected so far in semen or the vagina, curli and curli-like amyloid fibers are ubiquitous in mammalian hosts.(37) In fact, the innate immune response invoked by almost all amyloids has been proposed to have evolved as a response to curli amyloid formation by E. Coli.(38) Since functional amyloid fibers from bacteria or yeast similar to curli may be colocalized with $\mathrm{PAP}_{248-286}$ at the initial site of HIV infection, we tested the effect of preformed curli amyloid fibrils on the kinetics of SEVI fiber formation. We found curli does significantly enhance the rate of SEVI fibrillization, although through an unusual mechanism for a cross-seeding interaction.

\section{METHODS \& MATERIALS}

\section{Peptide preparation}

$\mathrm{PAP}_{248-286}$ was obtained from Biomatik Corporation. To prepare monomeric $\mathrm{PAP}_{248-286}$, lyophilized $\mathrm{PAP}_{248-286}$ was first quickly dissolved in $20 \%$ acetic acid to a final concentration of $1 \mathrm{mg} / \mathrm{ml}$ to remove preformed aggregates. The aggregate free solution was then lyophilized overnight. A $3 \mathrm{mg} / \mathrm{ml}$ stock solution was made from the lyophilized peptide in the assay buffer $(10 \mathrm{mM}$ phosphate buffer, $\mathrm{pH}$ 7.3 containing $150 \mathrm{mM} \mathrm{NaCl}$ ).

Human IAPP was obtained from Genscript. Monomeric IAPP was prepared by first dissolving in hexafluoroisopropanol to a concentration of $1 \mathrm{mg} / \mathrm{ml}$ then lyophilizing overnight. $\mathrm{A} \beta_{1-40}$ was obtained from Anaspec. Monomeric $A \beta_{1-40}$ was prepared in a similar way using $2 \%$ ammonium hydroxide instead of hexafluoroisopropanol. The lyophilized pellet of both peptides was dissolved directly in the assay buffer.

\section{CsgA and CsgB preparation}


CsgA and CsgB were expressed (39) and purified(40) as previously described. Briefly, both were overexpressed as His-tag fusion proteins in LSR12 bacteria. Following centrifugation for 20 min at $5,000 \mathrm{x} \mathrm{g}$, the bacterial pellet was incubated in $8 \mathrm{M}$ guanidine hydrochloride (from Sigma, adjusted to $\mathrm{pH} 7.2$ by the addition of $\mathrm{NaOH}$ ) for 24 hours with stirring. After incubation, the cells were centrifuged again for $20 \mathrm{~min}$ at $10,000 \mathrm{x}$ g and the supernatant sonicated. The solution was then passed over a HISSelect ${ }^{\mathrm{TM}} \mathrm{HF}$ nickel-nitrilotriacetic acid column, washing first with $50 \mathrm{mM} \mathrm{KPi}(\mathrm{pH} 7.3)$ followed by $12.5 \mathrm{mM}$ imidazole in $50 \mathrm{mM} \mathrm{KPi}(\mathrm{pH} \mathrm{7.3)}$ to remove non-specifically bound proteins and then with $125 \mathrm{mM}$ imidazole in $50 \mathrm{mM} \mathrm{KPi}(\mathrm{pH} 7.3)$ to elute CsgA or CsgB.

To prepare amyloid fibers of CsgA and $\mathrm{B}$, the curli containing fractions from the HIS-Select ${ }^{\mathrm{TM}}$ column allowed to fibrillize overnight at room temperature. Protein concentration was measured by the BCA (Bicinchoninic Acid) assay prior to fibrillization. The resulting fibers were then centrifuged (15 min at $10,000 \mathrm{x} \mathrm{g}$ ) and washed with water to remove the imidazole salt. This procedure was repeated four times and the pellet then lyophilized. The lyophilized pellets were then reconstituted in the assay buffer and sonicated with a probe sonicator (Sonic Dismembrator Model 100, Fischer Scientific) for approximately 1 minute prior to the aggregation assay.

Aggregation assays

Aggregation assays for $\mathrm{PAP}_{248-286}$ were performed in $10 \mathrm{mM}$ phosphate buffer, $\mathrm{pH} 7.3$ containing $150 \mathrm{mM} \mathrm{NaCl}$. A total volume of $40 \mu \mathrm{L}$ was placed in each well of a 96 well half area plate with a clear bottom, with $2 \mathrm{mg} / \mathrm{mL} \mathrm{PAP}_{248-286}, 25 \mu \mathrm{M}$ Thioflavin $\mathrm{T}$, and either $0,1,2.5$, or $5 \mathrm{~mol} \%$ preformed fibers of curli $A$ or $B$. Aggregation assays for IAPP and $A \beta_{1-40}$ were performed similarly except different concentrations of peptide $\left(2.5 \mu \mathrm{M}\right.$ of IAPP and $\left.5 \mu \mathrm{M} \mathrm{A} \beta_{1-40}\right)$ and CsgA and CsgB were used and the temperature for IAPP aggregation was set to $25^{\circ} \mathrm{C}$. Amyloid fibers of $\mathrm{A} \beta_{1-40}$ and IAPP were prepared by aggregation for 2 days at $37{ }^{\circ} \mathrm{C}$ as detailed below and were sonicated for 1 minute before loading onto the plate for the seeding experiments.

A single $1 \mathrm{~mm}$ glass bead was placed in each well to increase the aggregation rate and promote sample reproducibility.(41) The loaded plate was sealed, and placed on a BioTek Synergy 2 plate reader set at $37^{\circ} \mathrm{C}\left(\mathrm{PAP}_{248-286}\right.$ and $\left.\mathrm{A} \beta_{1-40}\right)$ or $25^{\circ} \mathrm{C}$ (IAPP) with a constant linear shaking speed of $17 \mathrm{~Hz}$. Absorbance was monitored at $350 \mathrm{~nm}$ and THT fluorescence measurements were taken with an excitation filter at $440 \mathrm{~nm}$ with a bandwidth of $30 \mathrm{~nm}$ and an emission filter at $485 \mathrm{~nm}$ with a bandwidth of $20 \mathrm{~nm}$. Data points were collected every 10 minutes, with continual shaking occurring between data points. All experiments were performed with samples in triplicate. The kinetic curves were fitted to a sigmoidal growth model that has empirically been found to reproduce most of the features of amyloid aggregation:

$$
\mathbf{I}=\frac{\mathrm{I}_{\mathrm{max}}-\mathrm{I}_{0}}{1+\mathrm{e}^{\left(\mathrm{t}-\mathrm{t}_{1 / 2}\right) \mathrm{f}}}
$$

Eq.1

where $I_{0}$ and $I_{\max }$ are the initial and maximum fluorescence or absorbance values, $t_{1 / 2}$ is the time required to reach half intensity, and the elongation time $t_{\mathrm{e}}$ is an apparent first order time constant for the addition of peptide to existing fibers. (42) The lag-time $t_{0}$ before detectable aggregation occurs is described by $\mathrm{t}_{0}=\mathrm{t}_{1 / 2}-2 \mathrm{t}_{\mathrm{e}}$.

\section{Transmission Electron Microscopy}

Samples of $6 \mu \mathrm{L}$ of $\mathrm{PAP}_{248-286}$ solutions after the aggregation experiment were applied to 200 mesh carbon coated copper electron microscopy grids and allowed to stand for 2 minutes. The grids were then washed with water 20 times to remove salts, after which $6 \mu \mathrm{L}$ of a $2 \%$ uranyl acetate solution 
was added and allowed to set for 1 minute before being removed. Fiber images were taken on a Phillips X-100 Transmission Electron Microscope operating at 60kV and 10,500x magnification.

\section{Cells for infectivity assays}

A CEM-GFP cell line that expresses a green fluorescent protein (GFP) driven by the HIV-1 LTR promoter was obtained through the AIDS Research and Reference Reagent Program, Division of AIDS, NIAID, NIH from Dr. Jacques Corbeil and maintained in RPMI1640 (Invitrogen) medium supplemented with 10\% heat-inactivated fetal bovine serum (HyClone) (RPMI-10) and containing 500 $\mu \mathrm{g} / \mathrm{ml}$ geneticin (Invitrogen).(43)

\section{Infectivity assays}

Infectivity assays were performed as previously described.(44) A viral stock solution was prepared by transfection of HeLa cells with a HIV-1 molecular clone pNL4-3. Supernatants of transfected cells were collected 2 days post-transfection, and viruses in the supernatant solution were pelleted by ultracentrifugation and resuspended in RPMI-10 medium. These virus stocks $(20,000 \mathrm{cpm}$ RT activity) were combined with stock solutions of SEVI amyloid fibers $(50 \mu \mathrm{g} / \mathrm{ml}$ from a stock solution of $440 \mu \mathrm{M} \mathrm{PAP}_{248-286}$ incubated under aseptic conditions for 7 days at $37{ }^{\circ} \mathrm{C}$ under vigorous agitation $(1,300 \mathrm{rpm})$ ) and used to inoculate $2 \times 10^{5}$ of CEM-GFP in $100 \mu \mathrm{l}$ RPMI-10 for 2 hours at 37 ${ }^{\circ} \mathrm{C}$. Cells were then washed and incubated in $1 \mathrm{ml}$ RPMI-10 at $37^{\circ} \mathrm{C}$. Inoculations were performed in triplicate. To block the second round of infection, the CD4 blocking antibody Leu3a $(0.25 \mu \mathrm{g} / \mathrm{ml})(\mathrm{BD}$ Biosciences) and the reverse transcriptase inhibitor AZT $(1 \mu \mathrm{M})($ Sigma) were added to the medium 12 hours post-infection. Two days post-infection, cells were fixed in $4 \%$ paraformaldehyde in PBS (phosphate buffer saline) and analyzed using a FACSCanto flow cytometer and the FlowJo software ver. 8.7.1. Efficiencies of infection were determined directly from the percentage of GFP positive cells after subtraction of the baseline activity obtained in the absence of HIV-1 $1_{\text {NL4-3. }}$ Comparisons between samples were made using a one-tailed unpaired Student t-test.

\section{RESULTS}

\section{Seeding with curli fibers greatly increases the rate of SEVI formation from PAP $248-286$}

To test the in vitro activity of curli on the kinetics of SEVI amyloid formation from $\mathrm{PAP}_{248-286}$, we measured the kinetics of amyloid formation and aggregation of $440 \mu \mathrm{M} \mathrm{PAP}_{248-286}$ solutions in the presence of curli nuclei.(41) The curli amyloid fiber is actually a composite of several proteins,(36) with CsgA serving as the main structural scaffold and CsgB nucleating amyloid formation from CsgA (sequences given in Fig. S1).(45)

PAP $_{248-286}$ aggregated slowly in the absence of preformed nuclei of any type under the conditions tested,(14) as shown by both turbidity measurements (a nonspecific indicator of general aggregation, Figs. 1C and 1D) and ThT fluorescence (a measurement specific for amyloid, Figs. 1A and 1B). The lag time of formation ( $60 \mathrm{hrs})$ is considerably longer than that previously described $(\sim 18 \mathrm{hrs}),(3,13)$ at an identical concentration, most likely due to a difference in shaking speed or ionic strength.(14)

Turbidity increased before ThT fluorescence for samples without CsgA or CsgB (Fig. 1). In addition, while the changes in turbidity could be closely approximated by a sigmoidal curve for all samples, analysis of the residuals from the sigmoidal fit to the ThT fluorescence shows two additional features not present in the turbidity curves. First, a second early component with a short lag time (about 18 hours, similar to previous observations $(3,13)$ ) but low ThT fluorescence (about $1 / 8$ of the final value) can be detected in the ThT measurements. Second, ThT fluorescence immediately decreases after 
the addition of high concentrations of $\mathrm{CsgA}$. These findings suggest amyloid formation by PAP ${ }_{248-286}$ may be a multistep process in which either the formation of non-amyloid prefibrillar aggregates occurs before amyloid formation for these samples $(46,47)$ or bundling of amyloid-like protofibrillar filaments is necessary for ThT binding to SEVI, as has been observed for other amyloidogenic proteins.(48)

The addition of preformed CsgA seed had a dramatic impact on the kinetics of SEVI amyloid formation. One mole \% CsgA (relative to $\mathrm{PAP}_{248-286}$ ) was sufficient to cause a six-fold decrease in the elongation time constant, which is reflective of the time for the addition of $\mathrm{PAP}_{248-286}$ to existing fibers (Fig. 2B). CsgA seeds had only a minor effect on the lag time (Figs. 2A and C). This is an unexpected result, as the addition of seeds usually results in the reduction or elimination of the lag-time with little corresponding change in the rate of addition to pre-existing fibers.(22) CsgB has qualitatively similar effects as CsgA on the kinetics of SEVI amyloid formation, however the magnitude of the effect is relatively less in comparison to CsgA (Figs. 1 and 2).

The difference in elongation rates for Csg initiated $\mathrm{PAP}_{248-286}$ fiber formation is reflected in the morphology of individual fibers. Fibers samples initiated by curli and those grown in their absence are morphologically similar, except for a large difference in the aspect ratios (Fig. 3A-C and Fig.S2). Standard SEVI fibers have an aspect ratio of approximately 5.8, while those grown with CsgA and $\mathrm{CsgB}$ are more heterogeneous and much longer, with aspect ratios of approximately 14.7 and 10.8 respectively (Fig. 3F and Fig. S2). This finding is in agreement with the greatly enhanced fiber elongation rate found in the presence of either of the curli fibers. The very short and broken fibers of all $\mathrm{PAP}_{248-286}$ samples are different than typical amyloid fibers, such as the CsgA and CsgB fibers formed under quiescent conditions (Fig. 3D and E.), most likely because the high degree of agitation required for SEVI fiber formation fragments nascent amyloid fibers.

\section{SEVI amyloid fibers obtained from curli nucleation retain the ability to enhance HIV infection}

We next confirmed that curli nucleated SEVI fiber samples retain a similar ability to promote HIV infection as SEVI fibers generated de novo. The activity of the SEVI fibrils incubated with Csg was tested using an infectivity assay in which a reporter $\mathrm{T}$ cell line that expresses GFP upon HIV infection was used.(43) In the absence of SEVI, flow cytometry showed a low percentage of GFPpositive cells, in agreement with the low infectivity of HIV under the conditions employed. The addition of SEVI fibrils generated de novo caused an approximately 8-fold increase in the infectivity (Fig. 4), roughly matching results of other studies of SEVI under conditions of high viral load. $(6,7,49)$ The much larger degree of enhancement shown in some studies $(>100,000$ times) is only apparent at very high viral dilution.(3) Neither the $\mathrm{PAP}_{248-286}$ monomer nor equivalent amounts of CsgA or CsgB alone had an effect on HIV infection efficiency (Fig 4). On the other hand, CsgA and CsgB nucleated fibers enhanced HIV infection at least to the same extent as de novo generated SEVI fibers (13 and 16 times respectively, Fig. 4). From these results, it can be seen that the SEVI fiber samples, regardless of how they were nucleated, show a comparable ability to enhance the rate of HIV infection.

Cross-seeding with curli at low concentrations affects the amyloidogenesis of other proteins besides $P A P_{248-286}$

The ability of curli to accelerate SEVI formation in the absence of any obvious sequence similarity suggests curli may accelerate amyloid formation by other proteins as well. To test this possibility, we performed analogous seeding experiments on the amyloidogenic peptides IAPP and A $\beta_{1 \text { - }}$ 40. Addition of preformed curli nuclei had a complex effect on the aggregation of both these peptides (Fig. 5 and Fig. S3). The fibrillization rate of IAPP was strongly decreased by low concentrations of both CsgA and Csg B (1\% of the IAPP concentration or $25 \mathrm{nM}$ ) (Fig. 5). Addition of CsgA, but not $\mathrm{CsgB}$, to IAPP also lowered the total ThT fluorescence, suggesting either fewer or shorter fibers are produced in the presence of CsgA. The effect of Csg A and CsgB on the fibrillization rate of $\mathrm{A} \beta_{1-40}$ was 
more modest, and showed a more complex dependence on the concentration with the fibrillization rate slightly decreasing at lower concentrations and slightly increasing at higher concentrations of both Csg A and CsgB (Fig. 5). The lag time of both peptides decreased by approximately $50 \%$ after addition of 10 mole \% of the oppositely charged Csg protein (CsgA for IAPP and CsgB for A $\beta_{1-40}$ ), smaller amounts had little effect. Addition of $1 \%$ of the similarly charged Csg protein increased the lag time of both peptides, surprisingly, larger amounts had little effect on the lag time. By comparison, addition of equivalent amounts of preformed $A \beta_{1-40}$ fibril seeds led to a linear decrease in induction time but had little effect on the fibrillization rate (Fig. S4). Although a complete characterization of curli with IAPP and $A \beta_{1-40}$ lies outside the scope of this study, it can be seen from these experiments that both the effect of curli nucleation on amyloid fibrillogenesis is not limited to $\mathrm{PAP}_{248-286}$ and that curli can serve as both an inhibitor and enhancer of fibrillization.

\section{DISCUSSION}

In this study, we characterized the kinetics of $\mathrm{PAP}_{248-286}$ cross-seeded by the curli proteins CsgA and $\mathrm{CsgB}$ in comparison to the analogous cross-seeding interactions with the more amyloidogenic proteins hIAPP and $A \beta_{1-40}$. The purpose of this experiment is two-fold. First, $\mathrm{PAP}_{248-286}$ is only biologically active in the SEVI amyloid fiber form. The production of these fibers is ultimately controlled by the rate of amyloidogenesis, as $\mathrm{PAP}_{248-286}$ is subject to inactivating proteolysis in its monomeric but not in its amyloid form. (12) Since SEVI fibers have been detected in semen under conditions that would apparently not easily allow aggregation in vitro of SEVI alone, $(3,9)$ extrinsic factors are a likely source to look for influences on $\mathrm{PAP}_{248-286}$ aggregation. Second, amyloid crossseeding as a general phenomenon is not well understood, as apparently contradictory results regarding the efficiency and specificity of cross-seeding have been obtained. (22-26, 28-31) In performing the cross-seeding of $\mathrm{PAP}_{248-286}$, we found that while the lag-time for amyloid formation is moderately affected by curli seeding, the elongation rate is greatly increased. This finding is novel for a crossseeding reaction and is discussed in more depth below.

An understanding of this result requires some knowledge of the basic mechanism of the crossseeding reaction. In epitaxial heteronucleation, growth occurs by specific structural matching of the seeding nucleus with the protein being seeded (Fig. 6).(21, 50) A greater understanding of this process can be made by considering the structural constraints for amyloid formation. The cross $\beta$-sheet structure common to all amyloids is built by the intermolecular association of $\beta$ sheets that are stabilized by hydrogen bonds between amide backbone atoms of adjacent sheets. Since the amide backbone, in contrast to the side-chain residues, is similar in all proteins, any unfolded or partially folded protein should theoretically be able to associate with preformed seeds of another to formed mixed fibers. However, amyloid fibers apparently derive much of their energy from the formation of a "steric zipper", in which the sidechains from adjacent sheets from an interlocking dry surface. $(21,51-53)$ It can be seen from this requirement that epitaxial heteronucleation is unlikely to occur if the seeding nucleus is structurally different then the protein being seeded, $(21,50,54)$ consistent with the observation that cross-seeding between amyloid proteins is most efficient when the two proteins have homologous sequences.(55) For most amyloidogenic proteins this requirement is quite strict and even single point mutations can eliminate the ability of one amyloidogenic protein to cross-seed another. For others, the best studied being $\alpha$-synuclein which seeds a variety of non-homologous proteins, this requirement is relaxed, possibly because the disorder present in the $\alpha$-synuclein's fiber structure can accommodate multiple fiber polymorphs and different fiber interfaces.(22)

In the simplest model of epitaxially nucleated amyloidogenesis, the elongation time decreases linearly with the concentration of the seed as each new seed provides a new point of fibril growth.(56, 57) However, the elongation time is less sensitive to the seed concentration in more complicated models 
explicitly considering secondary nucleation by fragmentation of existing fibers, as the new fibers created by fragmentation provide additional points for fibril growth that compete with the nuclei from the original seeds. $(57,58)$ The maximal elongation rate is in fact relatively insensitive to the seeding concentration when the number of nuclei created by fibril fragmentation is much greater than the number of nuclei available from seeding. $(57,58)$ Such behavior is typically observed experimentally $(22,23,56,59)$ and is seen here with the $A \beta_{1-40}$ protein (see fig. S4). Regardless of the exact quantitative expression, current models predict that the lag-time for epitaxial heteronucleation should be more strongly affected than the elongation time.

However, epitaxial heteronucleation is not the only possible mechanism by which cross-seeding can occur. In contrast to epitaxial heteronucleation, non-specific heteronucleation can affect both the lag-time and the elongation rate by affecting the stability of different species along the aggregation pathway or by lowering the surface tension associated with forming clusters of the protein. In these mechanisms, the seed is not necessarily incorporated into the new amyloid fiber. Non-specific heteronucleation has been proposed for surfaces and non-protein ligands,(60-62) but not to our knowledge for cross-seeding reactions with other amyloidogenic proteins. Experiments with Lys to Ala $\mathrm{PAP}_{248-286}$ mutants show that charge repulsion between monomer units destabilizes the amyloid fiber.(6) Lateral association of the curli fiber with the nascent $\mathrm{PAP}_{248-286}$ fiber can reduce this repulsion between monomer subunits, increasing the elongation rate but not affecting the lag-time. In this case, it is expected that the electrostatic differences between CsgA and CsgB ( -6 overall charge for CsgA and +5 for $\mathrm{CsgB}, \mathrm{PAP}_{248-286}$ carries an overall charge of +6 ) are primarily responsible for the difference in cross-seeding efficiencies, although differences atomic-level differences in the packing between CsgA and $\mathrm{CsgB}$ and $\mathrm{PAP}_{248-286}$ may also play a role. It is important to note that a stable interaction between the $\mathrm{PAP}_{248-286}$ amyloid fiber and CsgA or CsgB may not be required, as theoretical studies show that the stability of amyloid fibers increases with the length of the fiber.(63) A transient interaction of CsgA or $\mathrm{CsgB}$ with $\mathrm{PAP}_{248-286}$ may stabilize a short, energetically unfavorable $\mathrm{PAP}_{248-286}$ fiber long enough to promote the formation of a longer and more stable amyloid fiber.

The interaction of curli with IAPP and $A \beta_{1-40}$ is less clear. Electrostatics plays a role in crossseeding nucleation, as the oppositely charged curli protein reduces the lag-time of both IAPP and A $\beta_{1-40}$ but the similarly charged curli protein has less effect. Both $\mathrm{CsgA}$ and $\mathrm{CsgB}$ decrease the elongation rate of IAPP, most likely by binding to and blocking reactive fibril ends. The effect of CsgA and CsgB on the elongation rate of $A \beta_{1-40}$ has a complex concentration dependence, likely the result of a mixture of stimulatory and inhibitory effects previously observed in the binding of some ligands to amyloid peptides.

Although the effects of curli on $A \beta_{1-40}$ were moderate, cross-seeding between other bacterial functional amyloids and $A \beta_{1-40}$ may have greater clinical significance. For example, the bacteria Borrelia burgdorferi produces a curli-like amyloid protein that colocalizes with $\mathrm{A} \beta$ amyloid deposits in Alzheimer's patients.(64, 65) Similarly, inoculation with Chlamydia pneumoniae stimulates the production of $A \beta_{1-42}$ amyloid plaques, $(66,67)$ although to our knowledge a curli-like amyloid protein has not been found yet for Chlamydia pneumonia. Although a definitive link between bacterial infection and amyloid-associated neurodegenerative diseases has been elusive due to the difficulties in firmly establishing bacterial infection considering the low levels of bacteria typical of chronic long-term infections,(68) the identification of new amyloidogenic proteins in bacteria and mammals is increasing rapidly,(35) opening up the possibility of an increasing role for bacterial and viral infections in poorly understood amyloidogenic diseases.

ACKNOWLEDGMENT This study was supported by research funds from NIH (GM095640 to A.R., AI089282, to A.O., and AI073847 to M.R.C.). The following reagent was obtained through the AIDS 
Research and Reference Reagent Program, Division of AIDS, NIAID, NIH: CEM-GFP from Jacques Corbeil.

SUPPORTING INFORMATION AVAILABLE: Amino acid sequences of CsgA, CsgB and PAP $248-286$, kinetic traces of $A \beta_{1-40}$ and IAPP amyloid formation in the presence of CsgA and CsgB, lag times and elongation rates of $A \beta_{1-40}$ seeded by $A \beta_{1-40}$. 


\section{REFERENCES}

1. Thomas, J. A., D. E. Ott, and R. J. Gorelick. 2007. Efficiency of human immunodeficiency virus type 1 postentry infection processes: Evidence against disproportionate numbers of defective virions. J. Virol. 81:4367-4370.

2. Perelson, A. S., A. U. Neumann, M. Markowitz, J. M. Leonard, and D. D. Ho. 1996. HIV-1 dynamics in vivo: Virion clearance rate, infected cell life-span, and viral generation time. Science 271:1582-1586.

3. Munch, J., E. Rucker, L. Standker, K. Adermann, C. Goffinet, M. Schindler, S. Wildum, R. Chinnadurai, D. Rajan, A. Specht, G. Gimenez-Gallego, P. C. Sanchez, D. M. Fowler, A. Koulov, J. W. Kelly, W. Mothes, J. C. Grivel, L. Margolis, O. T. Keppler, W. G. Forssmann, and F. Kirchhoff. 2007. Semen-derived amyloid fibrils drastically enhance HIV infection. Cell 131:1059-1071.

4. Arnold, F., J. Schnell, O. Zirafi, C. Sturzel, C. Meier, T. Weil, L. Standker, W. G. Forssmann, N. R. Roan, W. C. Greene, F. Kirchhoff, and J. Munch. 2012. Naturally occurring fragments from two distinct regions of the prostatic acid phosphatase form amyloidogenic enhancers of HIV infection. J. Virol. 86:1244-1249.

5. Roan, N. R., J. A. Muller, H. C. Liu, S. Chu, F. Arnold, C. M. Sturzel, P. Walther, M. Dong, H. E. Witkowska, F. Kirchhoff, J. Munch, and W. C. Greene. 2011. Peptides released by physiological cleavage of semen coagulum proteins form amyloids that enhance HIV Infection. Cell Host Microbe 10:541-550.

6. Roan, N. R., J. Munch, N. Arhel, W. Mothes, J. Neidleman, A. Kobayashi, K. Smith-McCune, F. Kirchhoff, and W. C. Greene. 2009. The cationic properties of SEVI underlie its ability to enhance human immunodeficiency virus infection. J. Virol. 83:73-80.

7. Roan, N. R., S. Sowinski, J. Munch, F. Kirchhoff, and W. C. Greene. 2010. Aminoquinoline surfen inhibits the action of SEVI (Semen-derived Enhancer of Viral Infection). J. Biol. Chem. 285:1861-1869.

8. Wurm, M., A. Schambach, D. Lindemann, H. Hanenberg, L. Standker, W. G. Forssmann, R. Blasczyk, and P. A. Horn. 2010. The influence of semen-derived enhancer of virus infection on the efficiency of retroviral gene transfer. J. Gene. Med. 12:137-146.

9. Kim, K. A., M. Yolamanova, O. Zirafi, N. R. Roan, L. Standker, W. G. Forssmann, A. Burgener, N. Dejucq-Rainsford, B. H. Hahn, G. M. Shaw, W. C. Greene, F. Kirchhoff, and J. Muench. 2010. Semen-mediated enhancement of HIV infection is donor-dependent and correlates with the levels of SEVI. Retrovirology 7:55-67.

10. Capule, C. C., C. Brown, J. S. Olsen, S. Dewhurst, and J. Yang. 2012. Oligovalent amyloidbinding agents reduce SEVI-mediated enhancement of HIV-1 infection. J. Am. Chem. Soc. 134:905-908.

11. Olsen, J. S., C. Brown, C. C. Capule, M. Rubinshtein, T. M. Doran, R. K. Srivastava, C. Feng, B. L. Nilsson, J. Yang, and S. Dewhurst. 2010. Amyloid-binding small molecules efficiently block SEVI (semen-derived enhancer of virus infection)- and semen-mediated enhancement of HIV-1 infection. J. Biol. Chem. 285:35488-35496.

12. Martellini, J. A., A. L. Cole, P. Svoboda, O. Stuchlik, L. M. Chen, K. X. Chai, B. K. Gangrade, O. E. Sorensen, J. Pohl, and A. M. Cole. 2011. HIV-1 enhancing effect of prostatic acid phosphatase peptides is reduced in human seminal plasma. Plos One 6:e16285.

13. Ye, Z. Q., K. C. French, L. A. Popova, I. K. Lednev, M. M. Lopez, and G. I. Makhatadze. 2009. Mechanism of fibril formation by a 39-Residue Peptide (PAPf39) from human prostatic acidic phosphatase. Biochemistry 48:11582-11591. 
14. Olsen, J. S., J. T. M. DiMaio, T. M. Doran, C. Brown, B. L. Nilsson, and S. Dewhurst. 2012. Seminal Plasma accelerates semen-derived enhancer of viral infection (SEVI) fibril formation by the prostatic acid phosphatase (PAP(248-286)) peptide. J. Biol. Chem. 287:11842-11849.

15. Olzscha, H., S. M. Schermann, A. C. Woerner, S. Pinkert, M. H. Hecht, G. G. Tartaglia, M. Vendruscolo, M. Hayer-Hartl, F. U. Hartl, and R. M. Vabulas. 2011. Amyloid-like aggregates sequester numerous metastable proteins with essential cellular functions. Cell 144:67-78.

16. Manzoni, C., L. Colombo, P. Bigini, V. Diana, A. Cagnotto, M. Messa, M. Lupi, V. Bonetto, M. Pignataro, C. Airoldi, E. Sironi, A. Williams, and M. Salmona. 2011. The molecular assembly of amyloid $A \beta$ controls its neurotoxicity and binding to cellular proteins. PloS one 6:e24909.

17. Dzwolak, W., R. Jansen, V. Smirnovas, A. Loksztejn, S. Porowski, and R. Winter. 2005. Template-controlled conformational patterns of insulin fibrillar self-assembly reflect history of solvation of the amyloid nuclei. Phys. Chem. Chem. Phys. 7:1349-1351.

18. Foo, C. K., Y. Ohhashi, M. J. S. Kelly, M. Tanaka, and J. S. Weissman. 2011. Radically different amyloid conformations dictate the seeding specificity of a chimeric Sup35 prion. J. Mol. Biol. 408:1-8.

19. Cloe, A. L., J. P. R. O. Orgel, J. R. Sachleben, R. Tycko, and S. C. Meredith. 2011. The Japanese Mutant $A \beta(\Delta \mathrm{E} 22-\mathrm{A} \beta(1-39))$ forms fibrils instantaneously, with low-thioflavin $\mathrm{T}$ fluorescence: Seeding of Wild-Type $A \beta(1-40)$ into atypical fibrils by $\triangle E 22-A \beta(1-39)$. Biochemistry 50:2026-2039.

20. Chien, P., J. S. Weissman, and A. H. DePace. 2004. Emerging principles of conformation based prion inheritance. Annu. Rev. Biochem. 73:617-656.

21. Apostol, M. I., J. J. W. Wiltzius, M. R. Sawaya, D. Cascio, and D. Eisenberg. 2011. atomic structures suggest determinants of transmission barriers in mammalian prion disease. Biochemistry 50:2456-2463.

22. Yagi, H., E. Kusaka, K. Hongo, T. Mizobata, and Y. Kawata. 2005. Amyloid fibril formation of alpha-synuclein is accelerated by preformed amyloid seeds of other proteins - Implications for the mechanism of transmissible conformational diseases. J. Biol. Chem. 280:38609-38616.

23. Han, H. Y., P. H. Weinreb, and P. T. Lansbury. 1995. The core Alzheimers peptide NAC forms amyloid fibrils which seed and are seeded by beta-amyloid - Is NAC a common trigger or target in neurodegenerative disease? Chem. Biol. 2:163-169.

24. Westermark, P., K. Lundmark, and G. T. Westermark. 2009. Fibrils from designed non-amyloidrelated synthetic peptides induce aa-amyloidosis during inflammation in an animal model. PloS one 4:e6041.

25. Morales, R., L. D. Estrada, R. Diaz-Espinoza, D. Morales-Scheihing, M. C. Jara, J. Castilla, and C. Soto. 2010. Molecular cross talk between misfolded proteins in animal models of Alzheimer's and prion diseases. J. Neurosci. 30:4528-4535.

26. O'Nuallain, B., A. D. Williams, P. Westermark, and R. Wetzel. 2004. Seeding specificity in amyloid growth induced by heterologous fibrils. J. Biol. Chem. 279:17490-17499.

27. Ma, B. Y., and R. Nussinov. 2012. Selective molecular recognition in amyloid growth and transmission and cross-species barriers. J. Mol. Biol. 421:172-184.

28. MacPhee, C. E., and C. M. Dobson. 2000. Formation of mixed fibrils demonstrates the generic nature and potential utility of amyloid nanostructures. J. Am. Chem. Soc. 122:12707-12713.

29. Jarrett, J. T., and P. T. Lansbury. 1992. Amyloid fibril formation requires a chemically discriminating nucleation event - Studies of an amyloidogenic sequence from the bacterial protein Osmb. Biochemistry 31:12345-12352.

30. Hinz, J., L. M. Gierasch, and Z. Ignatova. 2008. Orthogonal cross-seeding: An approach to explore protein aggregates in living cells. Biochemistry 47:4196-4200. 
31. Rajan, R. S., M. E. Illing, N. F. Bence, and R. R. Kopito. 2001. Specificity in intracellular protein aggregation and inclusion body formation. Proc. Natl. Acad. Sci. U. S. A. 98:1306013065.

32. Yu, X., Y. Luo, P. Dinkel, J. Zheng, G. H. Wei, M. Margittai, R. Nussinov, and B. Y. Ma. 2012. Cross-seeding and conformational selection between three- and four-repeat human tau proteins. J. Biol. Chem. 287:14950-14959.

33. Siddiqua, A., Y. Luo, V. Meyer, M. A. Swanson, X. Yu, G. H. Wei, J. Zheng, G. R. Eaton, B. Y. Ma, R. Nussinov, S. S. Eaton, and M. Margittai. 2012. Conformational Basis for asymmetric seeding barrier in filaments of three- and four-repeat tau. J. Am. Chem. Soc. 134:10271-10278.

34. Thurman, A. R., and G. F. Doncel. 2010. Innate immunity and inflammatory response to trichomonas vaginalis and bacterial vaginosis: Relationship to HIV acquisition. Am. J. Reprod. Immunol. 65:89-98.

35. Jordal, P. B., M. S. Dueholm, P. Larsen, S. V. Petersen, J. J. Enghild, G. Christiansen, P. Hojrup, P. H. Nielsen, and D. E. Otzen. 2009. Widespread abundance of functional bacterial amyloid in mycolata and other gram-positive bacteria. Appl. Environ. Microbiol. 75:4101-4110.

36. Barnhart, M. M., and M. R. Chapman. 2006. Curli biogenesis and function. Annu. Rev. Microbiol. 60:131-147.

37. Cegelski, L., J. S. Pinkner, N. D. Hammer, C. K. Cusumano, C. S. Hung, E. Chorell, V. Aberg, J. N. Walker, P. C. Seed, F. Almqvist, M. R. Chapman, and S. J. Hultgren. 2009. Smallmolecule inhibitors target Escherichia coli amyloid biogenesis and biofilm formation. Nature Chem. Biol. 5:913-919.

38. Tukel, C., R. P. Wilson, J. H. Nishimori, M. Pezeshki, B. A. Chromy, and A. J. Baumler. 2009. Responses to amyloids of microbial and host origin are mediated through toll-like receptor 2. Cell Host Microbe 6:45-53.

39. Hammer, N. D., J. C. Schmidt, and M. R. Chapman. 2007. The curli nucleator protein, CsgB, contains an amyloidogenic domain that directs CsgA polymerization. Proc. Natl. Acad. Sci. U. S. A. 104:12494-12499.

40. Wang, X. A., D. R. Smith, J. W. Jones, and M. R. Chapman. 2007. In vitro polymerization of a functional Escherichia coli amyloid protein. J. Biol. Chem. 282:3713-3719.

41. Giehm, L., and D. E. Otzen. 2010. Strategies to increase the reproducibility of protein fibrillization in plate reader assays. Anal. Biochem. 400:270-281.

42. Naiki, H., K. Higuchi, K. Nakakuki, and T. Takeda. 1991. Kinetic analysis of amyloid fibril polymerization in vitro. Lab. Invest. 65:104-110.

43. Gervaix, A., D. West, L. M. Leoni, D. D. Richman, F. WongStaal, and J. Corbeil. 1997. A new reporter cell line to monitor HIV infection and drug susceptibility in vitro. Proc. Natl. Acad. Sci. U. S. A. 94:4653-4658.

44. Ono, A., K. Monde, and V. Chukkapalli. 2011. Assembly and replication of HIV-1 in T cells with low levels of phosphatidylinositol-(4,5)-bisphosphate. J. Virol. 85:3584-3595.

45. Wang, X., N. D. Hammer, and M. R. Chapman. 2008. The molecular basis of functional bacterial amyloid polymerization and nucleation. J. Biol. Chem. 283:21530-21539.

46. Suzuki, Y., J. R. Brender, K. Hartman, A. Ramamoorthy, and E. N. Marsh. Alternative pathways of human islet amyloid polypeptide aggregation distinguished by (19)F nuclear magnetic resonance-detected kinetics of monomer consumption. Biochemistry.In press.

47. Horvath, I., C. F. Weise, E. K. Andersson, E. Chorell, M. Sellstedt, C. Bengtsson, A. Olofsson, S. J. Hultgren, M. Chapman, M. Wolf-Watz, F. Almqvist, and P. Wittung-Stafshede. Mechanisms of protein oligomerization: inhibitor of functional amyloids templates alphasynuclein fibrillation. J. Am. Chem. Soc. 134:3439-3444.

48. Cabaleiro-Lago, C., I. Lynch, K. A. Dawson, and S. Linse. 2010. Inhibition of IAPP and IAPP((20-29)) fibrillation by polymeric nanoparticles. Langmuir 26:3453-3461. 
49. Hauber, I., H. Hohenberg, B. Holstermann, W. Hunstein, and J. Hauber. 2009. The main green tea polyphenol epigallocatechin-3-gallate counteracts semen-mediated enhancement of HIV infection. Proc. Natl. Acad. Sci. U. S. A. 106:9033-9038.

50. Wasmer, C., A. Zimmer, R. Sabate, A. Soragni, S. J. Saupe, C. Ritter, and B. H. Meier. 2010. structural similarity between the prion domain of HET-s and a homologue can explain amyloid cross-seeding in spite of limited sequence identity. J. Mol. Biol. 402:311-325.

51. Sawaya, M. R., S. Sambashivan, R. Nelson, M. I. Ivanova, S. A. Sievers, M. I. Apostol, M. J. Thompson, M. Balbirnie, J. J. W. Wiltzius, H. T. McFarlane, A. O. Madsen, C. Riekel, and D. Eisenberg. 2007. Atomic structures of amyloid cross-beta spines reveal varied steric zippers. Nature 447:453-457.

52. Eisenberg, D., S. A. Sievers, J. Karanicolas, H. W. Chang, A. Zhao, L. Jiang, O. Zirafi, J. T. Stevens, J. Munch, and D. Baker. 2011. Structure-based design of non-natural amino-acid inhibitors of amyloid fibril formation. Nature 475:96-U117.

53. Colletier, J. P., A. Laganowsky, M. Landau, M. L. Zhao, A. B. Soriaga, L. Goldschmidt, D. Flot, D. Cascio, M. R. Sawaya, and D. Eisenberg. 2011. Molecular basis for amyloid-beta polymorphism. Proc. Natl. Acad. Sci. U. S. A. 108:16938-16943.

54. Wiltzius, J. J. W., M. Landau, R. Nelson, M. R. Sawaya, M. I. Apostol, L. Goldschmidt, A. B. Soriaga, D. Cascio, K. Rajashankar, and D. Eisenberg. 2009. Molecular mechanisms for proteinencoded inheritance. Nat. Struct. Mol. Biol. 16:973-U998.

55. Krebs, M. R., L. A. Morozova-Roche, K. Daniel, C. V. Robinson, and C. M. Dobson. 2004. Observation of sequence specificity in the seeding of protein amyloid fibrils. Protein Sci. 13:1933-1938.

56. Padrick, S. B., and A. D. Miranker. 2002. Islet amyloid: Phase partitioning and secondary nucleation are central to the mechanism of fibrillogenesis. Biochemistry 41:4694-4703.

57. Cohen, S. I. A., M. Vendruscolo, C. M. Dobson, and T. P. J. Knowles. 2011. Nucleated polymerization with secondary pathways. II. Determination of self-consistent solutions to growth processes described by non-linear master equations. J. Chem. Phys. 135.

58. Knowles, T. P. J., C. A. Waudby, G. L. Devlin, S. I. A. Cohen, A. Aguzzi, M. Vendruscolo, E. M. Terentjev, M. E. Welland, and C. M. Dobson. 2009. An analytical solution to the kinetics of breakable filament assembly. Science 326:1533-1537.

59. Xue, W. F., S. W. Homans, and S. E. Radford. 2008. Systematic analysis of nucleationdependent polymerization reveals new insights into the mechanism of amyloid self-assembly. Proc. Natl. Acad. Sci. U. S. A. 105:8926-8931.

60. Pronchik, J., X. L. He, J. T. Giurleo, and D. S. Talaga. 2010. In vitro formation of amyloid from alpha-synuclein is dominated by reactions at hydrophobic interfaces. J. Am. Chem. Soc. 132:9797-9803.

61. Auer, S., A. Trovato, and M. Vendruscolo. 2009. A condensation-ordering mechanism in nanoparticle-catalyzed peptide aggregation. PLoS Comput. Biol. 5:e1000458.

62. Yin, J., R. Z. Chen, and C. L. Liu. 2009. Nucleic acid induced protein aggregation and its role in biology and pathology. Front. Biosci. 14:5084-5106.

63. Linse, B., and S. Linse. 2011. Monte Carlo simulations of protein amyloid formation reveal origin of sigmoidal aggregation kinetics. Mol. Biosyst. 7:2296-2303.

64. Miklossy, J. 2008. Chronic inflammation and amyloidogenesis in Alzheimer's disease - Role of spirochetes. J. Alzheimers Dis. 13:381-391.

65. Miklossy, J., K. Khalili, L. Gern, R. L. Ericson, P. Darekar, L. Bolle, J. Hurlimann, and B. J. Paster. 2004. Borrelia burgdorferi persists in the brain in chronic lyme neuroborreliosis and may be associated with Alzheimer disease. J. Alzheimers Dis. 6:639-649. 
66. Gerard, H. C., U. Dreses-Werringloer, K. S. Wildt, S. Deka, C. Oszust, B. J. Balin, W. H. Frey, E. Z. Bordayo, J. A. Whittum-Hudson, and A. P. Hudson. 2006. Chlamydophila (Chlamydia) pneumoniae in the Alzheimer's brain. FEMS Immunol. Med. Microbiol. 48:355-366.

67. Little, C. S., C. J. Hammond, A. MacIntyre, B. J. Balin, and D. M. Appelt. 2004. Chlamydia pneumoniae induces Alzheimer-like amyloid plaques in brains of BALB/c mice. Neurobiol. Aging 25:419-429.

68. Dobson, C. B., M. A. Wozniak, and R. F. Itzhaki. 2003. Do infectious agents play a role in dementia? Trends Microbiol. 11:312-317. 

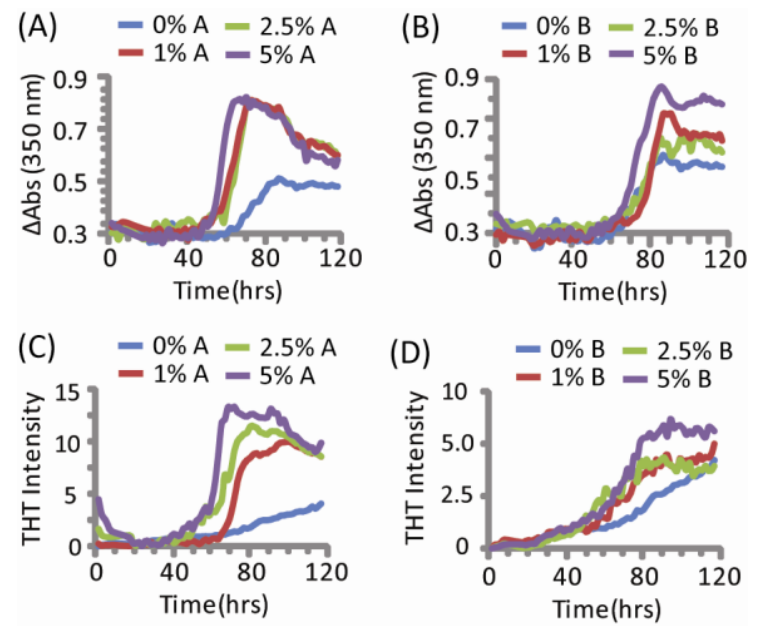

Figure 1. Kinetics of SEVI amyloid fiber formation in the presence of preformed fibers of CsgA and CsgB. Top: Turbidity measurements at $350 \mathrm{~nm}$ (A) $\mathrm{PAP}_{248-286}+\mathrm{Csg}$ A, (B) $\mathrm{PAP}_{248-286}+\mathrm{CsgB}$. Bottom: ThT fluorescence measurements of (C) $\mathrm{PAP}_{248-286}+\mathrm{CsgA}$, (D) $\mathrm{PAP}_{248-286}+\mathrm{Csg} \mathrm{B}$. Curves are averages for 3 measurements.
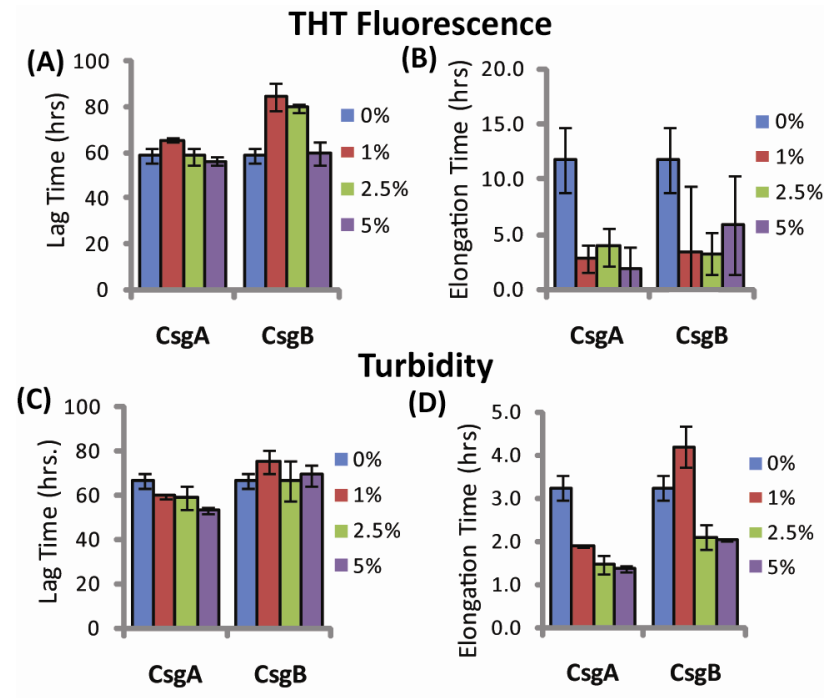

Figure 2. Elongation of SEVI amyloid fibers is significantly enhanced by preformed fibers of CsgA and $\mathrm{CsgB}$ lag time is less affected. Impact of preformed curli A and B fibers on the lag time (A and C) and elongation time (B and D) of SEVI formation. (A and B): Kinetic constants as determined by ThT fluorescence. (C and D): Kinetic constants as determined by turbidity measurements. Error bars represent S.E.M. 

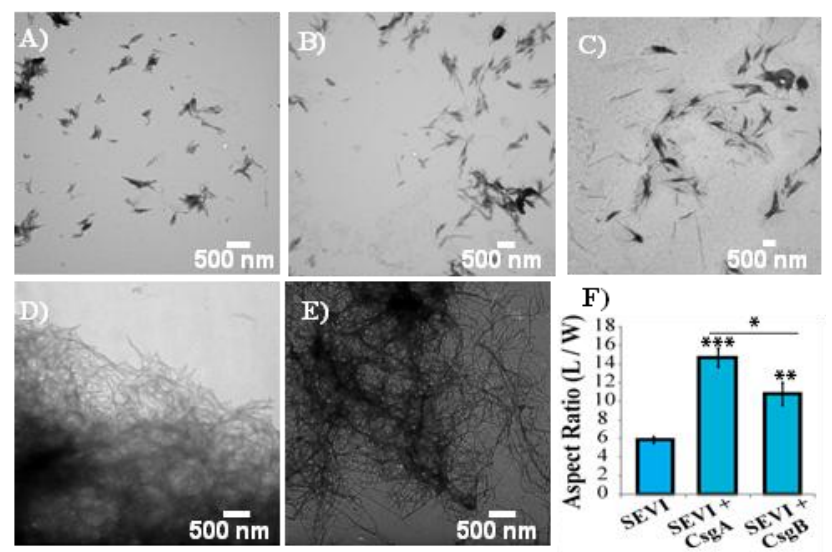

Figure 3. Curli nucleation produces longer SEVI fibers. Top: TEM images of SEVI fibers formed in the absence of curli (A) and in the presence of $5 \mathrm{~mol} \% \mathrm{CsgA}$ (B) and CsgB (C) fibers. Bottom: TEM images of CsgA (D) and CsgB (E) fibers. (F) Aspect ratios of individual fibers grown with and without curli nucleation ( $\mathrm{n}=$ 47, 23 and 37 for SEVI, SEVI+CsgA, and SEVI+CsgB respectively ). Fibers were grown at a concentration of $440 \mu \mathrm{M} \mathrm{PAP} 248-286$ at $37^{\circ} \mathrm{C}$ under $1400 \mathrm{rpm}$ orbital shaking for 7 days. $\mathrm{P}$ values were determined using a two-tailed unpaired Student t-test against the control sample.

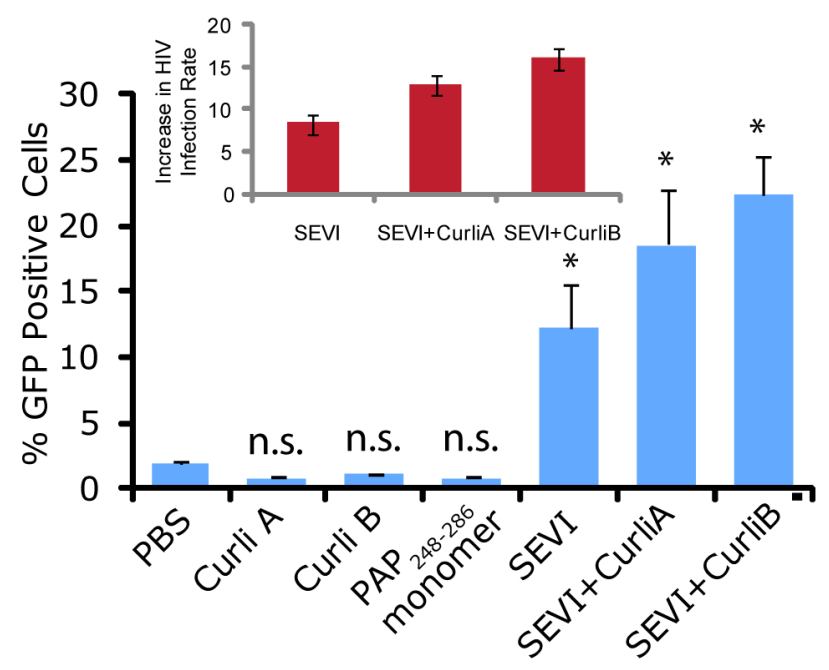

Figure 4. Curli nucleated SEVI fibers enhance HIV infectivity to a similar degree as SEVI generated de novo. CEM-GFP cells were infected with HIV $-1_{\text {NL4-3 }}$ (20,000 cpm RT activity) supplemented either with $50 \mu \mathrm{g} / \mathrm{ml}$ of the proteins indicated or PBS (phosphate buffered saline). SEVI samples were fibrillized for 7 days prior to infection. Measurements were performed in triplicate, error bars indicate S.E.M. P values were determined using a two-tailed unpaired Student t-test against the control sample. 
(A)
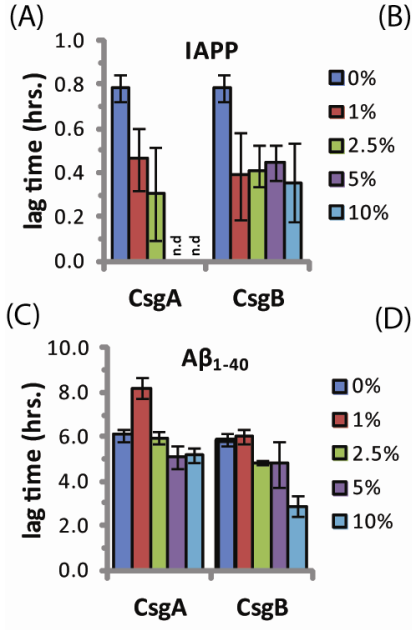

(B)

(D)
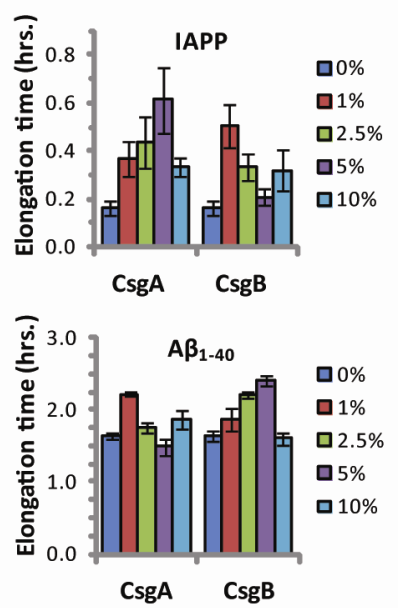

Figure 5. Curli's ability to influence amyloid formation is not limited to $\mathrm{PAP}_{248-286}$. Impact of preformed $\operatorname{csg} \mathrm{A}$ and $\operatorname{csgB}$ fibers on the lag time (A and $\mathrm{C}$ ) and elongation time (B and $\mathrm{D})$ of amyloid formation from $2.5 \mu \mathrm{M}$ IAPP (top) and $5 \mu \mathrm{M} \mathrm{A} \beta_{1-40}$ (bottom) as molar fractions of the IAPP and A $\beta_{1-40}$ concentrations.

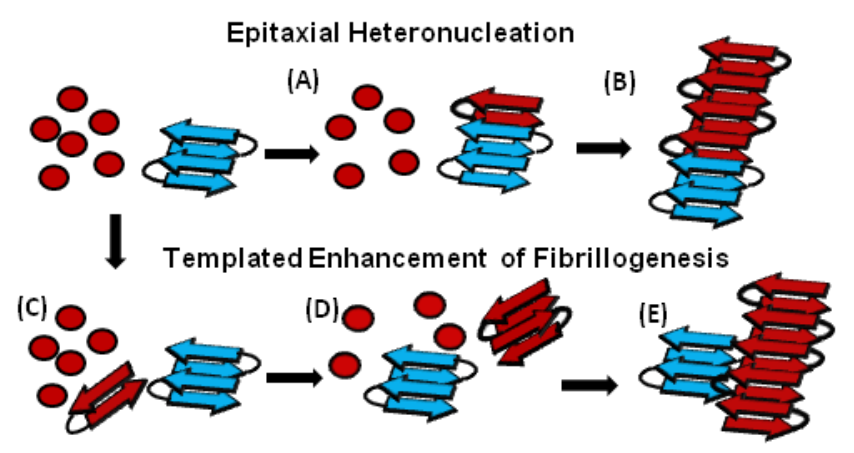

Figure 6. Cartoon models of possible cross-seeding reactions Top: Epitaxial Heteronucleation (A) Binding of the $\mathrm{PAP}_{248-286}$ monomer (red) to the curli seed (blue) induces formation of the $\beta$-sheet conformation of $\mathrm{PAP}_{248-286}$ (B) Fiber growth proceeds epitaxially from the seeding nucleus Bottom: Possible mechanism for non-epitaxial heteronucleation (C) A nucleus for the SEVI fiber forms independently of the curli fiber (D) Growth of the SEVI fiber initially proceeds slowly due to unfavorable interactions between subunits of the fiber (E) Lateral attachment of the nascent SEVI fiber to curli reduces repulsion between fiber subunits thereby enhancing the rate of fibrillogenesis. The curli seed may be incorporated into the final SEVI fiber or may detach to catalyze further fiber extension events. 


\section{Our Response to Reviewers' comments:}

Major changes in this version:

1. Distribution of height and width of fibers is included as new figure $\mathrm{S} 2$ in the supplementary material.

2. Kinetic measurements are analyzed in more depth including deviations from the sigmoidal model used to fit the data.

3. Modified the experimental section to include additional details as requested.

4. Fixed style inconsistancies to fit PeerJ format as noted.

\section{Reviewer Comments \\ Reviewer 1 \\ Basic reporting}

Several aspects of the author-guidelines have not been respected in the manuscript, particularly the citation guidelines have not been respected in the text. Please check the reference style section in the author-guidelines

\section{Minor comments:}

1. The year of Capule et al is missing in the text and the reference list P3 last paragraph: E. coli -> Escherichia coli

Response: E. coli has been expanded to Escherichia coli in its first appearance on page 3.

2. The use of italics for e.g. "in vivo" or "de novo" is not consistent throughout the text.

Response: "de novo", "in vivo", and "in vitro" have been italicized everywhere except the abstract where italics may cause errors with some indexing services such as Pubmed.

3. The abbreviations 'HIV' and 'SEVI' on line 2 and 3 in the abstract should be spelled out. Idem for IAPP, BCA...

Response: The abbreviations for HIV, SEVI, IAPP, and BCA have been spelled out at their first appearance.

4. P3 line 4: it is desirable to express the plural of 'an additional cofactor' (like the first line of the next paragraph) unless the authors have indications that only a single cofactor is appropriate.

Response: "Additional cofactor" has been changed to "additional cofactors"

\section{Experimental design}

The materials and methods section lacks some essential data and even incorrect information: some methods are described with insufficient detailed information to be fully reproducible by other investigators.

1. The source of $A \beta_{1-40}$ is not mentioned

Response: $A \beta_{1-40}$ was obtained from Anaspec, as noted in the revised manuscript.

2. $8 \mathrm{M}$ guanidinium $\mathrm{HCl}$ : which is the source of the chemical, and how was the $\mathrm{pH}$ controlled to $\mathrm{pH} 7.2$ ? 
Response: The $\mathrm{pH}$ was adjusted to 7.2 by titration with $\mathrm{NaOH}$. Guanidinium $\mathrm{HCl}$ was purchased from Sigma.

3. Was the protein concentration measured before or after the fibrillation?

Response: Protein concentration was measured before fibrillation, as the concentration of amyloid fibers is usually underestimated by most methods.

4. What do the authors mean with "prior to being loaded onto the plate"? It only becomes clear in the next paragraph if my interpretation is correct on how the experiment was actually performed.

Response: The phrase "prior to being loaded onto the plate" has been changed to "prior to the aggregation assay".

5. Fiber preparation of $A \beta_{1-40}$ should not be explained in the section "CsgA and CsgB preparation" but rather inserted in the next section "aggregation assays"

Response: The description of fiber preparation for IAPP and $A \beta_{1-40}$ has been moved to the next section.

6. Is the concentration of PAP $248-2863 \mathrm{mg} / \mathrm{mL}$ in the aggregation assay as indicated in the 'peptide preparation' section? Or is it $440 \mu \mathrm{M}(2 \mathrm{mg} / \mathrm{mL})$ as mentioned in the legend of Fig 3

Response: The concentration in the aggregation assay is $440 \mu \mathrm{M}(2 \mathrm{mg} / \mathrm{mL})$ as indicated. The stock solution prior to the addition of ThT and either buffer or fiber stock solution is 3 $\mathrm{mg} / \mathrm{ml}$ as indicated.

7. The ThT fluorescence is recorded with emission at $440 \mathrm{~nm}$ and excitation at $485 \mathrm{~nm}$. This is incorrect and actually impossible. Was the fluorescence measured at the top or bottom of the wells?

Response: The excitation is at $440 \mathrm{~nm}$ and the emission is at $485 \mathrm{~nm}$. This mistake has been fixed in the revised manuscript. Fluorescence was measured from the bottom of the wells due to the plastic seal on top used to prevent evaporation.

8. What is the operational voltage of the TEM analysis, and at which magnification were the images made?

Response: Images were acquired at $60 \mathrm{kV}$ and 10,500x magnification, as noted in the revised manuscript.

\section{Validity of the findings}

1. Is the non-seeded curve collected with the addition of buffer to the plate exactly the same manipulation as the addition of seeds?

Response: The control curve without seeds was treated and measured in the same way as the sample with seeds.

2. P6 line 6: can the authors rule out that the pretreatment with acetic acid explains the prolonged lag phase?

Response: Pretreatment with acetic acid is a standard treatment for disaggregating $\mathrm{PAP}_{248-}$ 286, and similar pretreatment with acid does not lead to a prolonged lag phase for other amyloidogenic proteins such as IAPP (see fig. S2). The brief exposure to acid is unlikely to 
make any post-translational modifications to the protein and acetic acid, being volatile, is easily removed by lyophilization.

3. That the turbidity increases prior to the increase in ThT fluorescence is difficult to interpret from figure 1, and seems not to be a valid statement for Fig 1B \& D. Also in Fig 2A \& C this does not seem to be significant.

4. Response: For SEVI samples without CsgA or CsgB, turbidity does change before ThT fluorescence. This can be seen clearly for these samples in Figs. 1 and 2. In Fig. 1 turbidity reaches a maximum at 80 hours while ThT fluorescence is still increasing after 120 hours. This is also a reflected in an increase in the elongation time in Fig. 2 (note that the scale is different in Fig. 2B and 2D to show clearly the difference between seeded and unseeded samples. Turbidity and ThT fluorescence increase roughly at the same time for SEVI samples with CsgA and Csg B.

To more accurately reflect this finding the sentence:

"Turbidity increased before ThT fluorescence, suggesting the formation of non-amyloid prefibrillar aggregates occurs before amyloid formation (Fig. 1).(1, 2) Non-fibrillar aggregates of $\mathrm{PAP}_{248-286}$ have been shown to have a similar effect on HIV infectivity as SEVI amyloid fibers.(3)

Has been changed to:

"Turbidity increased before ThT fluorescence for samples without CsgA or CsgB (Fig. 1). In addition, while the changes in turbidity could be closely approximated by a sigmoidal curve for all samples, analysis of the residuals from the sigmoidal fit to the ThT fluorescence shows two additional features not present in the turbidity curves. First, a second early component with a short lag time (about 18 hours, similar to previous observations $(3,4)$ ) but low ThT fluorescence (about 1/8 of the final value) can be detected in the ThT measurements. Second, ThT fluorescence immediately decreases after the addition of high concentrations of CsgA. These findings suggest amyloid formation by $\mathrm{PAP}_{248-286}$ may be a multistep process in which either the formation of non-amyloid prefibrillar aggregates occurs before amyloid formation for these samples $(1,2)$ or bundling of amyloid-like protofibrillar filaments is necessary for ThT binding to SEVI, as has been observed for other amyloidogenic proteins.(5)

5. The authors should specify more on how the non-seeded curve fits Equation 1. It seems that the transition in the cross-seeded ThT curve have an initial slow component followed by a cooperative aggregation behavior, while this is not mentioned or described by the authors.

Response: See answer to point 4.

6. Is there an explanation for the initial decrease in the ThT fluorescence (in the first 10-20h) with $2.5 \%$ and $5 \% \mathrm{CsgA}$ in Fig 1C?

Response: See answer to point 4. While removal of the first few points does improve the fit for these samples, it has little effect on the values calculated from the fit (56.3 vs 57.2 for the lag time and 2.3 vs. 2.6 for the elongation time for the $5 \% \mathrm{CsgA}$ sample with and without removal of the first points respectively).

7. The description of the fiber morphology is insufficient and should be extended with more details (dimensions, twists...). Aspects of fiber polymorphism and heterogeneity in the samples should also be described. The quality of the images is of insufficient quality / 
magnification to allow a careful assessment of the statements/interpretation of the authors. I find the absence of a characterization of the seeds a missed opportunity to strengthen this work. Are the "seeds" after sonication merely preformed fibers (see p4)? A simple TEM observation would clarify a lot.

Response: We recorded the height, width and aspect ratio distributions of the SEVI fibers (new Fig. S2). As seen below, all the samples are relatively heterogeneous, especially the cross-seeded samples with Csg A and Csg B. A further analysis of fiber morphology is difficult because the fibers are very short, which obscures interpretation of twists and branching, and heterogenous.

8. P6 4th paragraph: is the statement "Csg-containing SEVI fibrils" correct? Did the authors verify that the SEVI Fibrils resulting from the cross-seeding have incorporated Csg proteins in the fibrillar assembly?

Response: As noted in the Discussion section, it is difficult to verify if the low concentration of Csg seeds in the samples is actually incorporated into the SEVI fibers. As a result, the specific term "Csg-containing SEVI fibrils" has been replaced with the more general term "SEVI fibrils incubated with Csg".

9. p7 3rd sentence of the discussion: this statement is contradictory to the last sentence of 1 st paragraph of page 6

Response: The statement "The production of these fibers is ultimately controlled by the rate of amyloidogenesis, as $\mathrm{PAP}_{248-286}$ is subject to inactivating proteolysis in its monomeric but not in its amyloid form" on pg 6 may appear to be in contradiction with the statement on pg7 "Non-fibrillar aggregates of $\mathrm{PAP}_{248-286}$ have been shown to have a similar effect on HIV infectivity as SEVI amyloid fibers". However, it is likely, although currently unproven, that proteolysis of monomeric $\mathrm{PAP}_{248-286}$ will also lead to reduction of oligomeric but not fibrillar forms of SEVI, since it has been shown that similar interactions are frequently involved in oligomeric and fibrillar forms of amyloidogenic peptides.

\section{Comments for the author}

Although I acknowledge the potential in the presented study, I have concerns about the description of the materials and methods and the interpretation of the data. Particularly the materials and methods section should be optimized significantly to allow other investigators to reproduce this work.

More care should have been used while preparing the manuscript for the present submission.

\section{Reviewer 2}

\section{Basic reporting}

No comments

\section{Experimental design}

No comments

\section{Validity of the findings}

No comments

\section{Comments for the author}

This is a very interesting paper which clearly illustrates the complex cross-seeding/elongation effects. I completely agree with the authors: cross-seeding among amyloids with different 
sequences is common; and like the authors, I expect it is much more common that previously recognized. This aspect has been recently reviewed (e.g. Selective molecular recognition in amyloid growth and transmission and cross-species barriers. J. Mol. Biol. 2012; 421(2-3):17284 ), and emphasized again with respect to tau proteins (Cross-seeding and conformational selection between three- and four-repeat human Tau proteins (J. Biol. Chem. 2012; 287(18):14950-9; Conformational basis for asymmetric seeding barrier in filaments of three- and four-repeat tau. J. Am. Chem. Soc. 2012 Jun 20;134(24):10271-8). The authors may wish to relate to some of the references noted above which discuss this behavior for a different amyloid species. However, what is of particular interest in this work is that it experimentally goes much beyond these: it shows the complex behavior of sequence species: it may moderately affect the nucleation rate while significantly enhance the growth of fibers from existing nuclei; or it may decrease the lag-time of amyloid formation but strongly inhibit elongation; or have concentration effects, all likely depending on the details sequence matching, and broadly on the environment, including concentration.

Altogether, this work points to an important, yet overlooked aspect of amyloidosis and 'infectivity': cross-seeding is there, and is a reflection of the conformational heterogeneity of amyloids, monomers and oligomers. Further, it can also be expressed by heterogeneous consequences, which can be expected. On the downside, it also points to the difficulties in designing drugs to inhibit amyloid formation.

This work is novel and highly significant, and should be published in PeerJ, and I hope without delay. I expect it would be well-cited, and followed by other such studies, along similar lines. The manuscript can be accepted as is.

Response: We appreciate the reviewer's positive response and have included the references suggested.

1. Suzuki, Y., J. R. Brender, K. Hartman, A. Ramamoorthy, and E. N. Marsh. Alternative Pathways of Human Islet Amyloid Polypeptide Aggregation Distinguished by (19)F Nuclear Magnetic Resonance-Detected Kinetics of Monomer Consumption. Biochemistry.

2. Horvath, I., C. F. Weise, E. K. Andersson, E. Chorell, M. Sellstedt, C. Bengtsson, A. Olofsson, S. J. Hultgren, M. Chapman, M. Wolf-Watz, F. Almqvist, and P. Wittung-Stafshede. Mechanisms of Protein Oligomerization: Inhibitor of Functional Amyloids Templates alpha-Synuclein Fibrillation. J. Am. Chem. Soc. 134:3439-3444.

3. Munch, J., E. Rucker, L. Standker, K. Adermann, C. Goffinet, M. Schindler, S. Wildum, R. Chinnadurai, D. Rajan, A. Specht, G. Gimenez-Gallego, P. C. Sanchez, D. M. Fowler, A. Koulov, J. W. Kelly, W. Mothes, J. C. Grivel, L. Margolis, O. T. Keppler, W. G. Forssmann, and F. Kirchhoff. 2007. Semen-derived amyloid fibrils drastically enhance HIV infection. Cell 131:1059-1071.

4. Ye, Z. Q., K. C. French, L. A. Popova, I. K. Lednev, M. M. Lopez, and G. I. Makhatadze. 2009. Mechanism of Fibril Formation by a 39-Residue Peptide (PAPf39) from Human Prostatic Acidic Phosphatase. Biochemistry 48:11582-11591.

5. Cabaleiro-Lago, C., I. Lynch, K. A. Dawson, and S. Linse. 2010. Inhibition of IAPP and IAPP((2029)) Fibrillation by Polymeric Nanoparticles. Langmuir 26:3453-3461. 


\section{Figure 1}

Kinetics of SEVI amyloid fiber formation in the presence of preformed fibers of CsgA and CsgB.
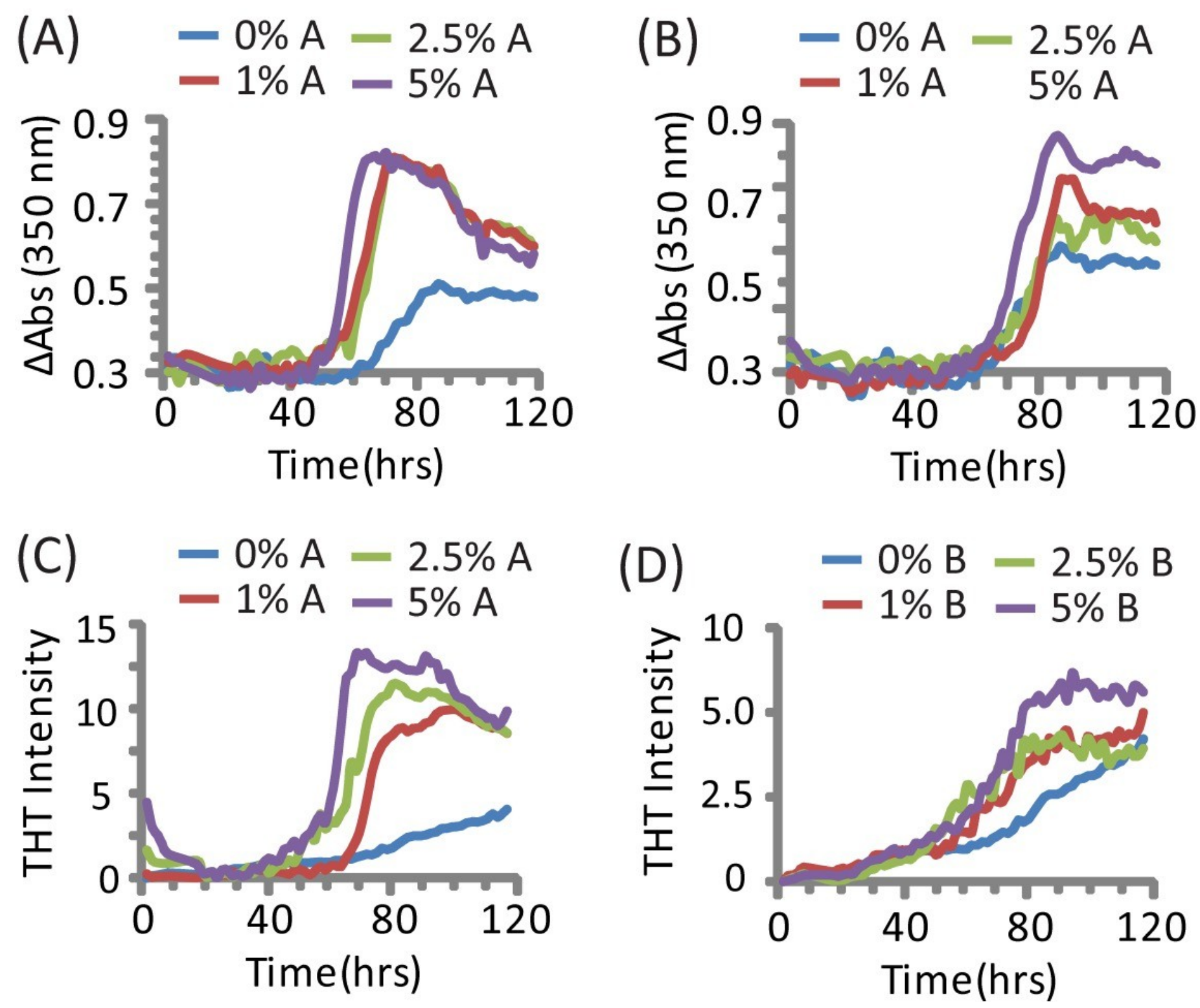


\section{Figure 2}

Elongation of SEVI amyloid fibers is significantly enhanced by preformed fibers of CsgA and CsgB lag time is less affected

\section{THT Fluorescence}

(A)

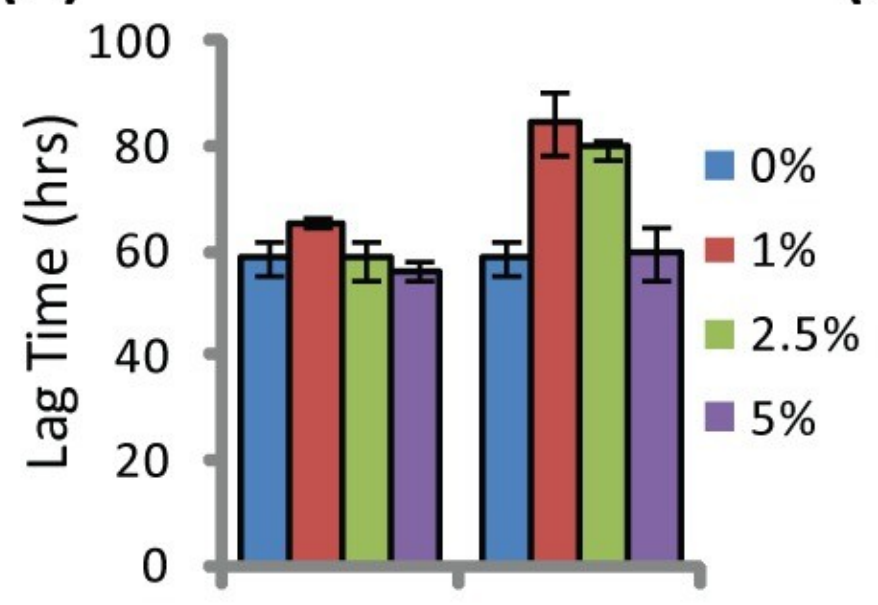

CsgA

(C)

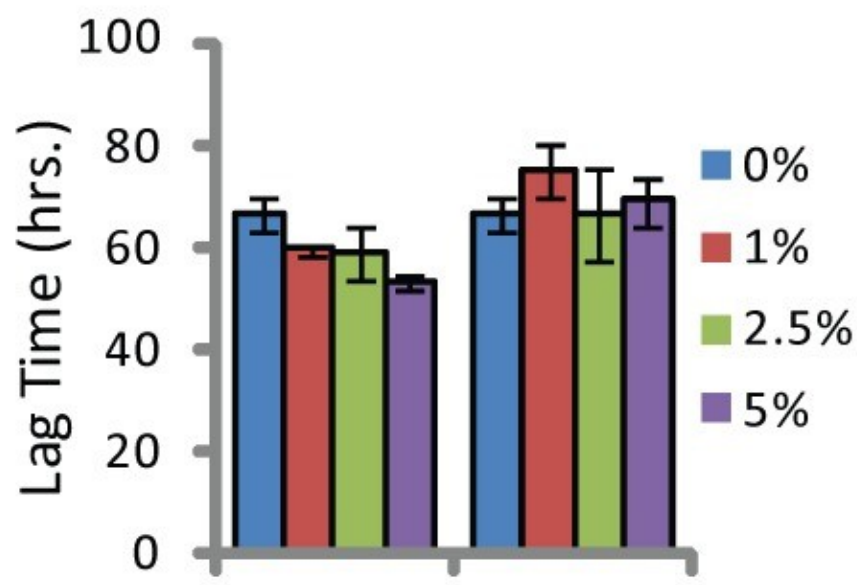

CsgA
CsgB

Turbidity

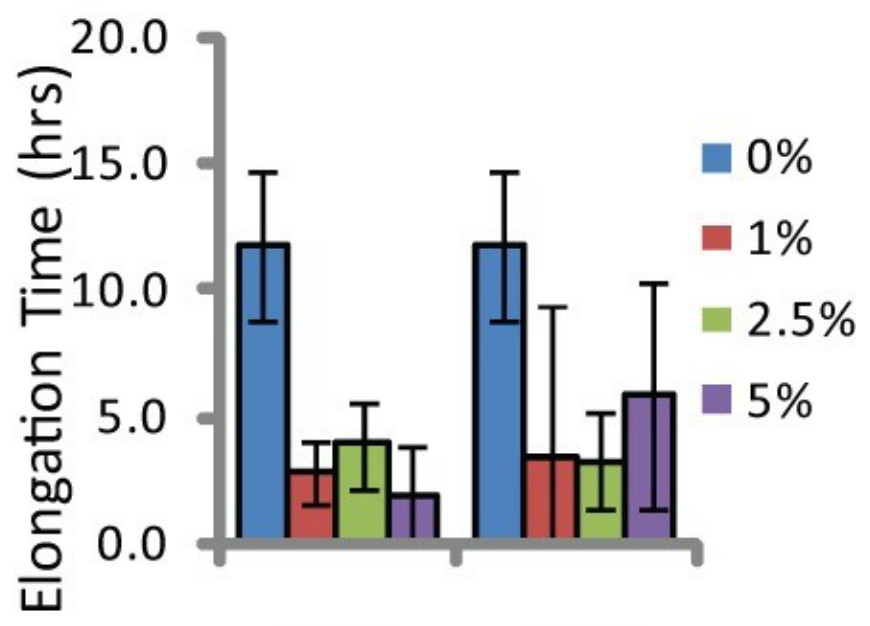

CsgA

(D) 


\section{Figure 3}

Curli nucleation produces longer SEVI fibers.

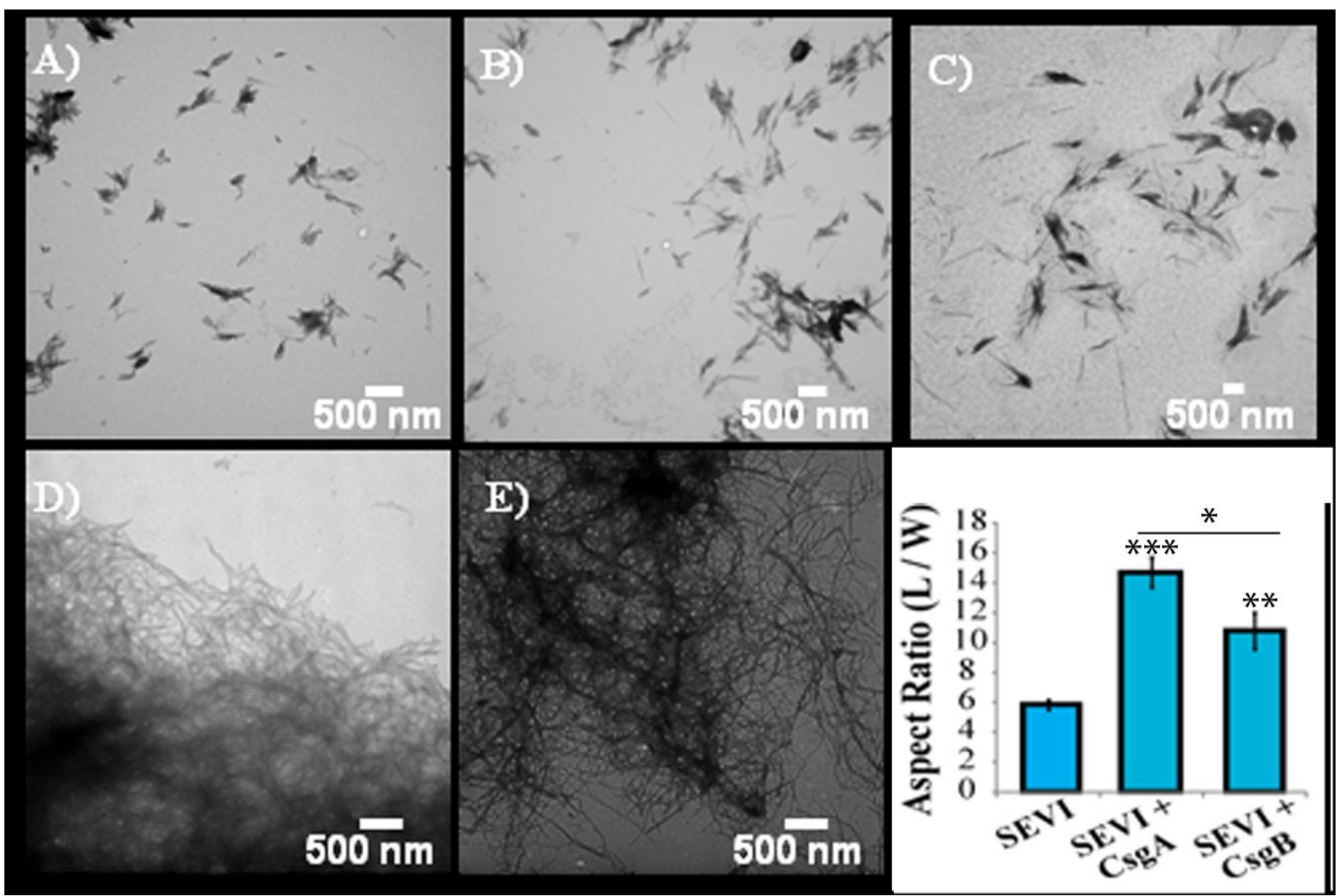


Figure 4

Curli nucleated SEVI fibers enhance HIV infectivity to a similar degree as SEVI generated de novo.

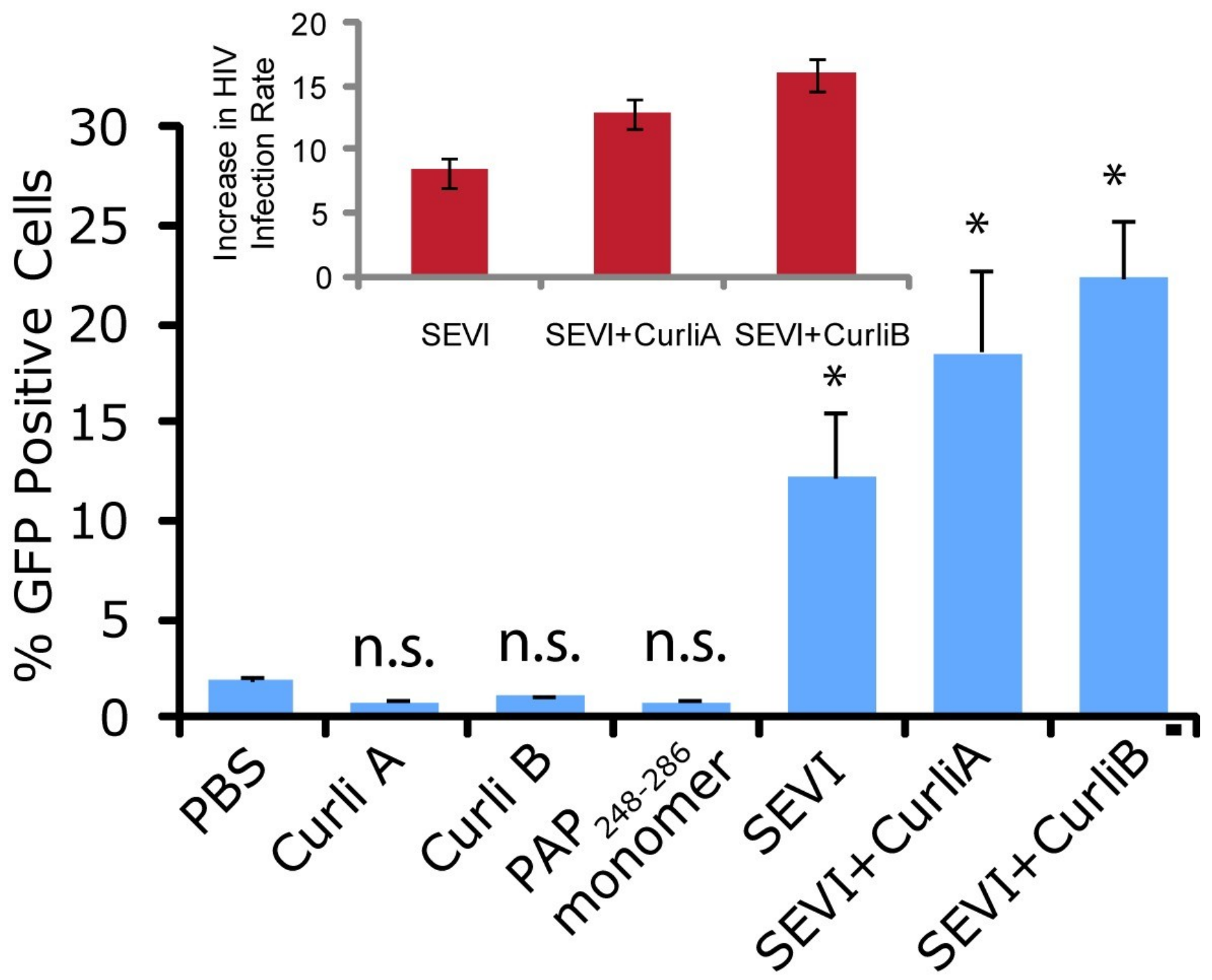




\section{Figure 5}

Curli's ability to influence amyloid formation is not limited to PAP 248-286 .

(A)

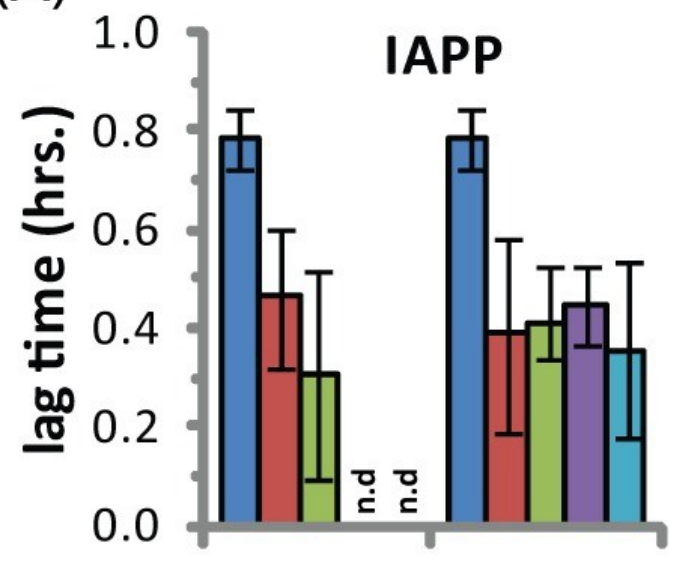

(C)

CsgA CsgB

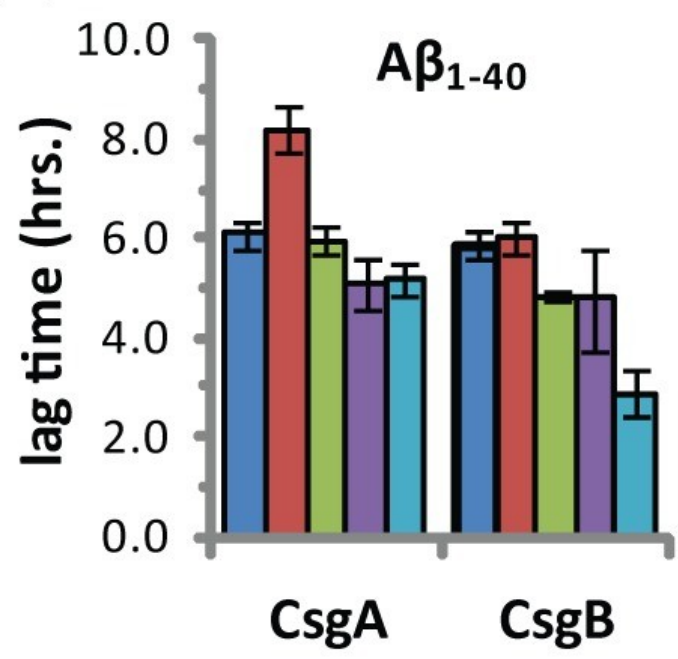

(B)

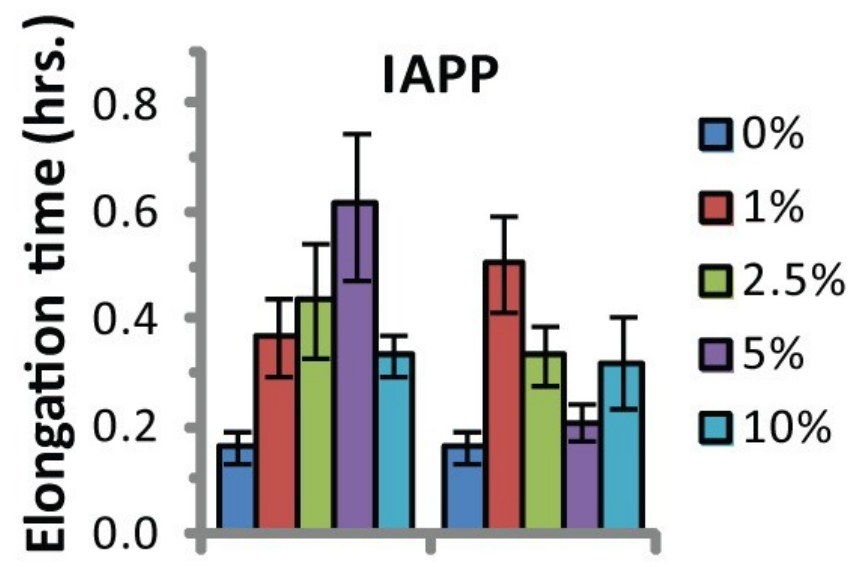

(D)

CsgA CsgB

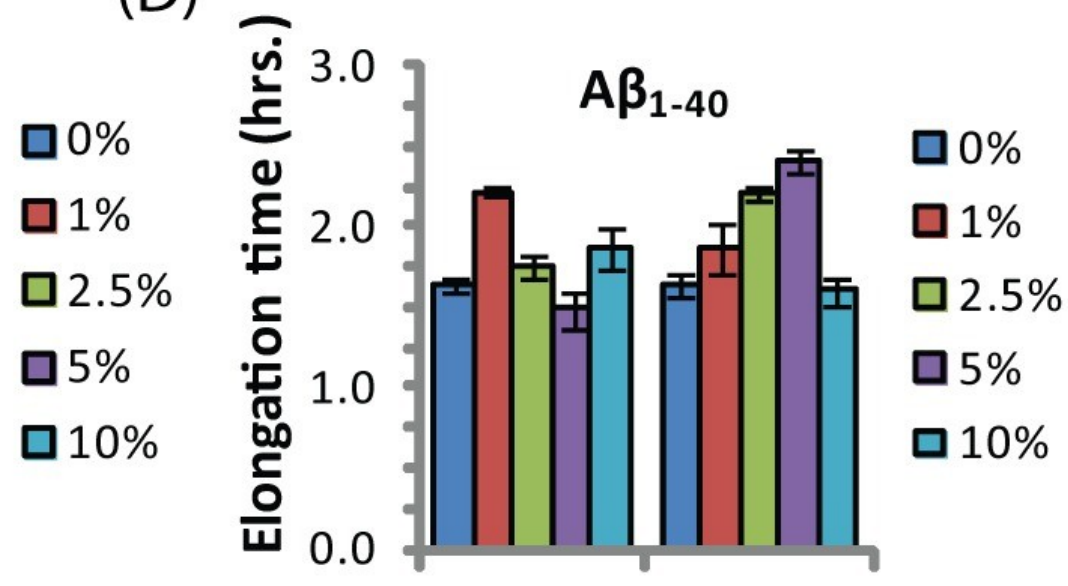

CsgA CsgB 
Figure 6

Cartoon models of possible cross-seeding reactions

Epitaxial Heteronucleation

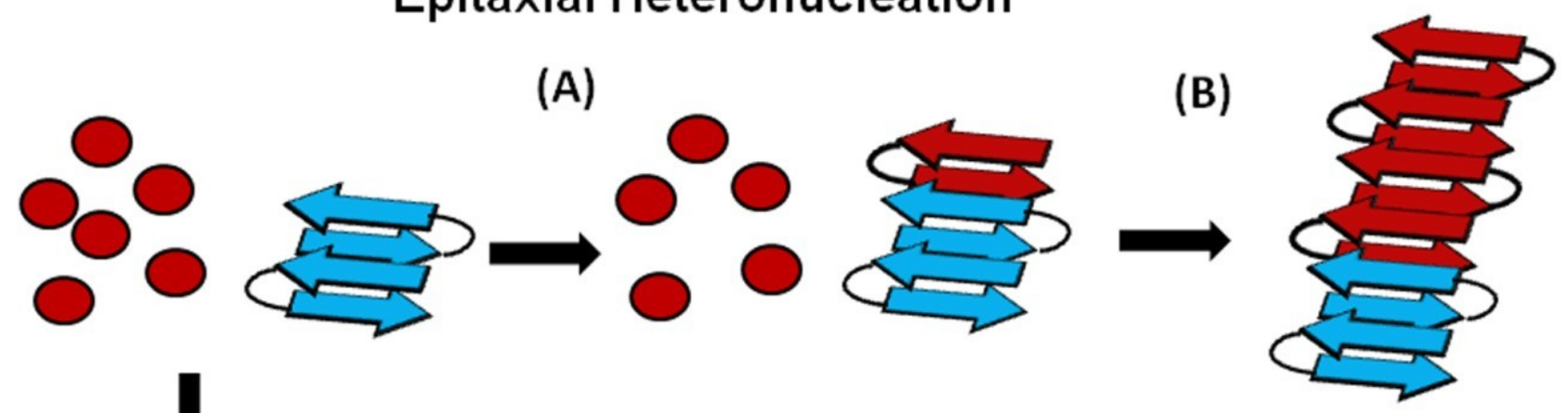

Templated Enhancement of Fibrillogenesis

(C)

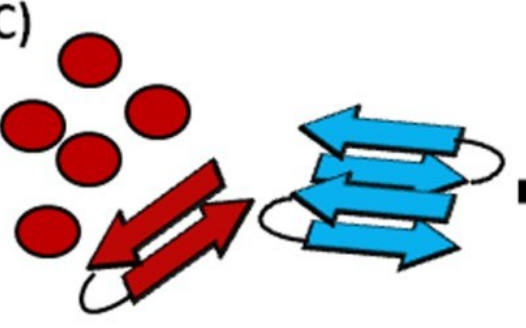

(D)

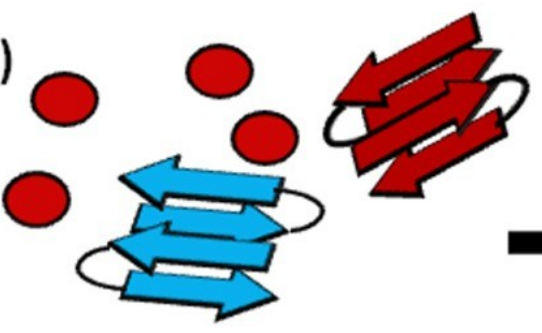

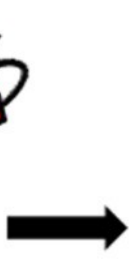

(E)

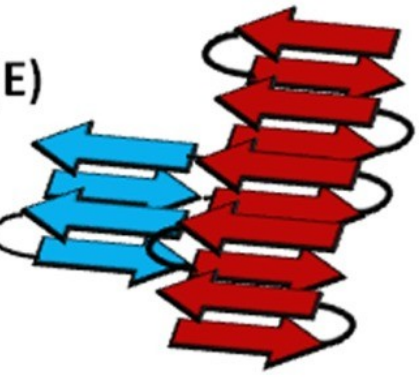

